\title{
HIGHER PRODUCTS
}

\author{
BY \\ GERALD J. PORTER( $\left.{ }^{1}\right)$
}

The study of secondary product operations has played an important role in algebraic topology. Among examples of such operations are the toral construction of Toda [9] and the secondary product of Massey [4]. The first of these has always been defined topologically while the second has been defined algebraically. This difference has been carried over to higher order products where higher Toda brackets have been studied by Gershenson [1]; and higher Massey products, by Kraines [3]. Even in Spanier's general setting [7] Toda brackets are defined in a topological category and Massey products in an algebraic category.

Massey products may, however, be defined in homotopy terms by means of a spectrum. In this paper we present a unified study of higher product operations in homotopy theory. This gives higher Massey products and higher Toda brackets as examples of the same general construction. It also extends the notion of Massey product to extraordinary cohomology theories with associative products.

A similar construction is given for the commutator product in a loop space and higher commutator products are defined. It is shown that these are related to the generalized higher order Whitehead products defined in [5].

Given a set of based topological spaces, $\left\{R_{j}\right\}_{j \in J}$, we say an associative pairing, $\mu: R_{i} \times R_{j} \rightarrow R_{i j}\left(R_{i j}=R_{k}\right.$ some $\left.k \in J\right)$, is a product if $\mu(x, *)=\mu(*, y)=*$, where $*$ is used ambiguously to denote the respective basepoints.

Such a product induces an external product

$$
\left[X, R_{i}\right] \times\left[Y, R_{j}\right] \rightarrow\left[X \times Y, R_{i j}\right]
$$

and an internal product

$$
\left[X, R_{i}\right] \times\left[X, R_{j}\right] \rightarrow\left[X, R_{i j}\right]
$$

where $[X, Y]$ is the set of based homotopy classes of maps $X \rightarrow Y$.

For homotopy classes $\alpha_{1} \in\left[X_{1}, R_{i}\right], \alpha_{2} \in\left[X_{2}, R_{j}\right]$, and $\alpha_{3} \in\left[X_{3}, R_{k}\right]$ a secondary external product $\left\{\alpha_{1}, \alpha_{2}, \alpha_{3}\right\} \in\left[\wedge\left(X_{1}, X_{2}, X_{3}\right), \Omega R_{i j k}\right]$ is defined whenever the external products $\alpha_{1} \alpha_{2}$ and $\alpha_{2} \alpha_{3}$ are zero. ( $\bigwedge=$ smash product, $\Omega=$ loop space).

If $X_{1}=X_{2}=X_{3}=X$ and the internal products $\alpha_{1} \alpha_{2}$ and $\alpha_{2} \alpha_{3}$ are zero, a secondary internal product $\left\langle\alpha_{1}, \alpha_{2}, \alpha_{3}\right\rangle \in\left[X, \Omega R_{i j k}\right]$ is defined.

Received by the editors August 2, 1968.

(1) The author was supported in part by NSF GP-6969.

Copyright (C) 1970, American Mathematical Society 
More generally an $(n-1)$ ary external product

$$
\left\{\alpha_{1}, \ldots, \alpha_{n}\right\} \in\left[\bigwedge\left(X_{1}, \ldots, X_{n}\right), \Omega^{n-2} R\left(i_{1}, \ldots, i_{n}\right)\right]
$$

and an $(n-1)$ ary internal product

$$
\left\langle\alpha_{1}, \ldots, \alpha_{n}\right\rangle \in\left[X, \Omega^{n-2} R\left(i_{1}, \ldots, i_{n}\right)\right]
$$

are defined whenever certain lower order products vanish.

The external products are generalizations of the secondary products in stable homotopy and the internal products are generalizations of the higher Massey products.

The construction is by means of a universal example and the same construction defines both products. We begin in $\$ 1$ by giving some definitions and-notation. The higher products are then defined in $\$ 2$.

The properties of the higher products are studied in $\S 3$. It is shown that they are natural on the left with respect to any map (Theorem 3.1) and natural on the right with respect to morphisms of ringed sets (Theorem 3.2). If $\left[R_{i}, R_{i}\right]$ is a group, multiplication by an integer can be defined. It is shown that the products are linear with respect to this multiplication (Corollary 3.4). An associativity relation between higher products is stated and proven in Theorem 3.7. This relation enables us to view higher products as operations derived from higher associativity and to compute the ambiguity introduced in the last step of the construction (Theorem 3.9.). It is well known that cup products are zero in suspensions. Similarly the higher internal products vanish on suspensions (Theorem 3.10). From this it follows that the higher internal products are annihilated by loop suspension (Corollary 3.12). External products, on the other hand, are of great interest in suspensions. In Theorem 3.14 we show that the external products commute with suspension in an appropriate sense. Finally a relationship between the primary product and the higher product is given in Theorem 3.16.

As a particular application of our construction we are able to define Massey products in any cohomology theory arising from an associative ringed spectrum. In $\$ 4$ we define "cochains" for such a theory. Roughly speaking, cochains correspond to null-homotopies. If $h$ is a null-homotopy of $f$, the coboundary $\delta$ is defined by $\delta h=f$. Thus cocycles correspond to null-homotopies of the constant map, i.e. loops. Using these ideas we show that formally our definitions coincide with the definition of higher Massey products given in [3].

The construction given in $\$ 2$ requires strict associativity of the product. If the pairing is only homotopy associative, the analogue of Stasheff's $A_{n}$ forms [8] are required. We study this problem in $\S 5$ and indicate how to define $n$-fold products given a ringed set with $A_{n}$ forms.

Finally, in $\S 6$ we consider the commutator product in a loop space. In this case 
the products are nonassociative but do satisfy a Jacobi identity. A construction similar to the one made in $\$ 2$ is given and higher commutator products are defined. Again there are internal and external products. The external product is a generalization of the Samelson product and is adjoint to the generalized Whitehead product defined in [5]. Four definitions are given of higher commutivity of a loop space, $\Omega X$. If $X$ is an $H$-space these all agree; however, in general the precise relationship between these definitions is unknown.

1. Preliminaries. We assume throughout that all spaces have base points, denoted $*$, and all maps are continuous and base point preserving. The set of maps $f: X \rightarrow Y$ will be denoted by $\{X, Y\}$ and the set of homotopy classes of maps $X \rightarrow Y$ will be denoted $[X, Y]$.

The cartesian product, $X \times Y$, is defined as usual. $X \vee Y$ is the subset of $X \times Y$ with at least one coordinate at a base point. The smash product

$$
\bigwedge(X, Y)=(X \times Y) /(X \vee Y) \text {. }
$$

The iterated smash $\wedge\left(X_{1}, \ldots, X_{n}\right)$ is defined inductively to be

$$
\bigwedge\left(\bigwedge\left(X_{1}, \ldots, X_{n-1}\right), X_{n}\right)
$$

We note that any other grouping, e.g. $\bigwedge\left(\bigwedge\left(X_{1}, \ldots, X_{j}\right), \bigwedge\left(X_{j+1}, \ldots, X_{n}\right)\right)$, is homeomorphic to $\bigwedge\left(X_{1}, \ldots, X_{n}\right)$.

DefinItion 1.1. We say that $\mathscr{R}=\left\{R_{j}, \mu\right\}_{j \in J}$ is a ringed set of topological spaces if each $R_{j}$ is a topological space with base point and for certain distinguished pairs $(i, j) \in J \times J, \mu: R_{i} \times R_{j} \rightarrow R_{k}$ (some $k \in J$ ) is a continuous map which sends $R_{i} \vee R_{j}$ to $*$.

In general we shall write $R(i, j)$ for the range of the multiplication on $R_{i} \times R_{j}$. Assume inductively that we have defined "distinguished $k$-tuple" for $k \leqq n-1$. We say an $n$-tuple, $\left(R_{j_{1}}, \ldots, R_{j_{n}}\right)$, is distinguished if the $(n-1)$-tuples, $\left(R_{j_{1}}, \ldots, R_{j_{n-1}}\right)$ and $\left(R_{j_{2}}, \ldots, R_{j_{n}}\right)$, are distinguished and the pairs, $\left(R\left(j_{1}, \ldots, j_{n-1}\right), R_{j_{n}}\right)$ and $\left(R_{j_{1}}, R\left(j_{2}, \ldots, j_{n}\right)\right)$, are distinguished. If $\left(R_{j_{1}}, \ldots, R_{j_{n}}\right)$ is a distinguished $n$-tuple we insist that all possible iterated multiplications have $R\left(j_{1}, \ldots, j_{n}\right)$ as their common range.

DEFINITION 1.2. $\mathscr{R}$ is said to be associative if the following diagram commutes for all distinguished 3-tuples $(i, j, k)$ and homotopy associative if the diagram homotopy commutes.

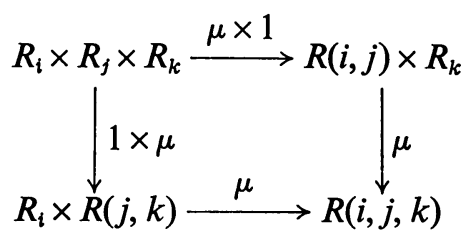


EXAMPLES. 1. $\mathscr{R}=\left\{S^{p}, \mu\right\}_{p \in Z^{+}}$, all pairs are distinguished, and $\mu: S^{p} \times S^{q} \rightarrow S^{p+q}$ is the quotient map. This pairing yields the stable Toda product.

2. $\mathscr{R}=F\left(\left\{X_{i}\right\}_{i \in I}\right)$, the free ringed set generated by $\left\{X_{i}\right\}_{i \in I}$, is $\left\{X\left(i_{1}, \ldots, i_{n}\right)\right.$ $\left.=\bigwedge\left(X_{i_{1}}, \ldots, X_{i_{n}}\right)\right\}$ all pairs are distinguished and $\mu$ is the quotient map.

3. $\mathscr{R}=\{K(\pi, m), \mu\}$ where $K(\pi, m)$ is an Eilenberg-MacLane space, $\pi$ a commutative ring with $1 .\left(K(\pi, m), K\left(\pi^{\prime}, n\right)\right)$ is distinguished if there is a pairing $\pi \otimes \pi^{\prime} \rightarrow \pi^{\prime \prime}$ for some $\pi^{\prime \prime}$. Then $\mu: K(\pi, m) \times K\left(\pi^{\prime}, n\right) \rightarrow K\left(\pi^{\prime \prime}, m+n\right)$ is the cup product. In general $\mu$ may not be strictly associative, however a model may be chosen for $K(\pi, n)$ and $\mu$ such that $\mu$ is associative. If $\mu$ is only homotopy associative then for all $n$ there exist $A_{n}$ forms for $\mu$. (See $\$ 5$ for definitions of $A_{n}$ forms.) This pairing yields the classical Massey product.

4. $\mathscr{R}=\left\{X^{Y}\right\}$ where $X^{Y}$ is the space of continuous base point preserving maps, $Y \rightarrow X$, with the compact open topology. A pair $\left(B^{A}, D^{C}\right)$ is distinguished if $C=B$ and, in this case, $\mu: B^{A} \times D^{B} \rightarrow D^{A}$ is composition. This pairing gives rise to the classical Toda bracket.

5. Given a ringed set $\mathscr{R}$ define $\Omega \mathscr{R}$ to be the set consisting of $\Omega^{j} R_{m}$ for $j \geqq 0$ and $R_{m} \in \mathscr{R}$. The pairing $\bar{\mu}: \Omega^{j} R_{m} \times \Omega^{k} R_{n} \rightarrow \Omega^{j+k} R(m, n)$ is given by

$$
\bar{\mu}\left(x_{1}, x_{2}\right)\left(t_{1}, \ldots, t_{j+k}\right)=\mu\left(x_{1}\left(t_{1}, \ldots, t_{j}\right), x_{2}\left(t_{j+1}, \ldots, t_{j+k}\right)\right)
$$

where $x_{1} \in \Omega^{j} R_{m}, x_{2} \in \Omega^{k} R_{n}$ and (for this definition only) we consider $\Omega^{j} R_{m}$ as the set of base point preserving maps $\left(I^{j}, I^{j}\right) \rightarrow\left(R_{m}, *\right)$ and $\mu$ is the pairing given in $\mathscr{R}$. Clearly $\Omega \mathscr{R}$ is a ringed set. $(\Omega \mathscr{R})^{k}$ is the ringed set consisting of $\Omega^{j} R_{m}$ for $j \geqq k$ and $\bar{\mu}$ as above.

6. Given a ringed set $\mathscr{R}$ define $\Sigma \mathscr{R}$ to be the set consisting of $\Sigma^{j} R_{m}$ for $j \geqq 0$ and $R_{m} \in \mathscr{R}$. The pairing $\bar{\mu}: \Sigma^{j} R_{m} \times \Sigma^{k} R_{n} \rightarrow \Sigma^{j+k} R(m, n)$ is the composite

$$
\Sigma^{j} R_{m} \times \Sigma^{k} R_{n} \rightarrow \bigwedge\left(\Sigma^{j} R_{m}, \Sigma^{k} R_{n}\right) \rightarrow \Sigma^{j+k} \bigwedge\left(R_{m}, R_{n}\right) \rightarrow \Sigma^{j+k} R(m, n)
$$

where the last map is induced by the pairing in $\mathscr{R}$. Clearly $\Sigma \mathscr{R}$ is a ringed set. $(\Sigma \mathscr{R})^{k}$ is the ringed set consisting of $\Sigma^{j} R_{m}$ for $j \geqq k$ and $\bar{\mu}$ as above.

7. For any ringed set $\mathscr{R}$ there is an associated ringed set $\bigvee \mathscr{R}$ of matrices of $\mathscr{R}$. The elements of $\bigvee \mathscr{R}$ are $m n$-fold wedges of spaces in $\mathscr{R}$. We denote

$$
\bigvee_{1 \leqq i \leqq m ; 1 \leqq j \leqq n} R_{i j}
$$

by $\left\{R_{i j}\right\}_{m, n}$. A pair, $\left(\left\{R_{i j}\right\}_{m, n},\left\{R_{i j}^{\prime}\right\}_{p, q}\right)$, is distinguishẹd if

(a) $p=n$,

(b) $\left(R_{i j}, R_{j k}^{\prime}\right)$ is distinguished in $\mathscr{R}$ for all $i, j, k$,

(c) $\mu: R_{i j} \times R_{j k}^{\prime} \rightarrow R_{i k}^{\prime \prime}$ for all $i, j, k$. 
Define $\bar{\mu}:\left\{R_{i j}\right\}_{m, n} \times\left\{R_{i j}^{\prime}\right\}_{n, p} \rightarrow\left\{R_{i j}^{\prime \prime}\right\}_{m, p}$, if the pair is distinguished, by

$$
\begin{aligned}
\bar{\mu}(x, y) & =\mu(x, y) \in R_{i k}^{\prime \prime} \quad \text { if }(x, y) \in R_{i j} \times R_{j k}^{\prime} \text { for some } i, j, k, \\
& =* \text { otherwise. }
\end{aligned}
$$

It is easily seen that this is well defined and $\bigvee \mathscr{R}$ is an associative ringed set if $\mu$ is associative. The matrix Toda bracket in stable homotopy defined by J. M. Cohen [11] arises from this pairing.

8. Let $\mathscr{R}$ be a ringed set in which each $R_{i} \in \mathscr{R}$ is an $H$-space with sum $\sigma_{i}: R_{i} \times R_{i} \rightarrow R_{i}$. The product $\mu: R_{i} \times R_{j} \rightarrow R(i, j)$ is said to be distributive with respect to $\sigma_{i}$ if

$$
\left.\mu\left(\sigma_{i}(x, y), z\right)\right)=\sigma_{i j}(\mu(x, z), \mu(y, z)) \quad \text { and } \quad \mu\left(x, \sigma_{j}(y, z)\right)=\sigma_{i j}(\mu(x, y), \mu(x, z))_{j} .
$$

The set $\Pi \mathscr{R}$ of matrices is defined to be the set of $m n$-fold products of elements of $\mathscr{R}$. Denote $\prod_{1 \leqq i \leqq m ; 1 \leqq j \leqq n} R_{i j}$ by $\left[R_{i j}\right]_{m, n}$. A pair, $\left(\left[R_{i j}\right]_{m, n},\left[R_{i j}^{\prime}\right]_{p, q}\right)$, is distinguished if it satisfies conditions (a), (b), and (c) of the preceding example. Let $\left(\sigma_{i}\right)^{n}:\left(R_{i}\right)^{n} \rightarrow R_{i}$ be some $n$-fold iteration of $\sigma_{i}$. On distinguished pairs the product

$$
\bar{\mu}:\left[R_{i j}\right]_{m, n} \times\left[R_{i j}^{\prime}\right]_{n, p} \rightarrow\left[R_{i j}^{\prime \prime}\right]_{m, p}
$$

is defined to be the composite

$$
\prod_{i, j} R_{i j} \times \prod_{k, l} R_{k l}^{\prime} \rightarrow \prod_{i, j, k} R_{i j} \times R_{j k}^{\prime} \rightarrow \prod_{i, k} \prod_{j} R_{i k}^{\prime \prime} \rightarrow \prod_{i, k} R_{i k}^{\prime \prime}
$$

where the first map is the product of projections, the second map is induced by the product $\mu$ in $\mathscr{R}$ and the third map is the product of $n$-fold summations. It is easily seen that $\bar{\mu}(x, *)=\bar{\mu}(*, y)=*$. If $\left(\mu, \sigma_{i}\right)$ is distributive, $\sigma_{i}$ is associative and abelian, and $\mu$ is associative then $\bar{\mu}$ is associative. If $\mathscr{R}$ is the ringed set described in Example 3 , the products arising from $\Pi \mathscr{R}$ are the matric Massey products studied by J. P. May [12].

9. If $G$ is a topological group or an associative $H$-space then the product $\mu: G \times G \rightarrow G$ is not a product in our sense since $\mu(*, 1) \sim 1$. This operation should more properly be called a sum. However the commutator product is a product in our sense but it is not associative. (Higher commutator products are studied in §6.)

Let $I^{n}$ denote the cartesian product of the unit interval with itself $n$ times. The boundary of $I^{n}$ is denoted by $I^{n} . U^{n} \subset I^{n}$ and $L^{n} \subset I^{n}$ are defined by

$$
\begin{aligned}
U^{n} & =\left\{\left(t_{1}, \ldots, t_{n}\right) \mid t_{i}=1 \text { for some } i\right\}, \\
L^{n} & =\left\{\left(t_{1}, \ldots, t_{n}\right) \mid t_{i}=0 \text { for some } i\right\}
\end{aligned}
$$

$U^{n}$ is the set of upper faces and $L^{n}$ the set of lower faces. Obviously $U^{n} \approx L^{n} \approx I^{n-1}$.

The path space of $X$ is denoted by $P X$ and the loop space by $\Omega X$. We take as our model for $P \Omega^{n-1} X$ the set of maps, $f:\left(I^{n}, L^{n}\right) \rightarrow(X, *) ; \Omega^{n} X$ is the set of maps $f:\left(I^{n+1}, L^{n+1}\right) \rightarrow(X, *)$. Each of these sets is given the compact open topology and the constant map is chosen as base point. 
Let $f: A \rightarrow P \Omega^{n-1} X$. For $1 \leqq i \leqq n$ we define $f^{i}: A \rightarrow P \Omega^{n-2} X$ by restriction to the $i$ th upper face, i.e.

$$
f^{i}(a)\left(t_{1}, \ldots, t_{n-1}\right)=f(a)\left(t_{1}, \ldots, t_{i-1}, 1, t_{i}, \ldots, t_{n-1}\right) .
$$

Similarly if $f: A \rightarrow \Omega^{n} X$, for $1 \leqq i \leqq n+1$ we define $f^{i}: A \rightarrow P \Omega^{n-1} X$ by

$$
f^{i}(a)\left(t_{1}, \ldots, t_{n}\right)=f(a)\left(t_{1}, \ldots, t_{i-1}, 1, t_{i}, \ldots, t_{n}\right) .
$$

We say $g_{1}, \ldots, g_{n+1} \in\left\{A, P \Omega^{n-1} X\right\}$ are compatible if $\left(g_{j}\right)^{i}=\left(g_{i}\right)^{j-1}$ for $i<j$. In this case we define

$$
G=\sum_{i=1}^{n+1}(-1)^{i+1} g_{i} \in\left\{A, \Omega^{n} X\right\}
$$

by setting

$$
G(a)\left(t_{1}, \ldots, t_{i-1}, 1, t_{i+1}, \ldots, t_{n+1}\right)=g_{i}(a)\left(t_{1}, \ldots, t_{i-1}, t_{i+1}, \ldots, t_{n+1}\right)
$$

and $G(a) \mid L^{n+1}=*$. The compatibility conditions ensure that $G$ is well defined. This is addition in the sense of the homotopy addition theorem.

If $\left(R_{m}, R_{n}\right)$ is distinguished the product pairing induces the following pairings

$$
\begin{aligned}
&\left\{X, P \Omega^{j-1} R_{m}\right\} \times\left\{X, P \Omega^{k-1} R_{n}\right\} \rightarrow\left\{X, P \Omega^{j+k-1} R(m, n)\right\}, \\
&\left\{X, P \Omega^{j-1} R_{m}\right\} \times\left\{X, R_{n}\right\} \rightarrow\left\{X, P \Omega^{j-1} R(m, n)\right\}, \\
&\left\{X, R_{m}\right\} \times\left\{X, P \Omega^{j-1} R_{n}\right\} \rightarrow\left\{X, P \Omega^{j-1} R(m, n)\right\} .
\end{aligned}
$$

This is given in the first case by setting

$$
(f \cdot g)(x)\left(t_{1}, \ldots, t_{j+k}\right)=\theta_{2}(m, n)\left(f(x)\left(t_{1}, \ldots, t_{j}\right), g(x)\left(t_{j+1}, \ldots, t_{j+k}\right)\right)
$$

and in the other cases by similar formulae. If $\left(t_{1}, \ldots, t_{j+k}\right) \in L^{j+k}$ then either $\left(t_{1}, \ldots, t_{j}\right) \in L^{j}$ or $\left(t_{j+1}, \ldots, t_{j+k}\right) \in L^{k}$. In either case $(f \cdot g)(x)\left(t_{1}, \ldots, t_{j+k}\right)=*$ and $f \cdot g$ lies in the desired set. These products correspond to the definition of cup product on the cochain level. (For details see $\S 4$.)

2. Construction of the higher products-Associative case. The $n$-fold universal product is defined in a universal example. The construction of the universal example is conceptually easy; however, there are many definitions to be made and details to be checked $\left({ }^{2}\right)$. The universal example corresponding to the distinguished $n$-tuple $\left(R_{j_{1}}, \ldots, R_{j_{n}}\right)$, is denoted $E_{n}\left(j_{1}, \ldots, j_{n}\right)$. To define this we make use of intermediate spaces $E_{i}\left(j_{1}, \ldots, j_{n}\right), 2 \leqq i<n$, which may be thought of as universal examples for $i$-fold products in $n$ variables. In particular, $E_{2}\left(j_{1}, \ldots, j_{n}\right)$ is $R_{j_{1}} \times \cdots \times R_{j_{n}}$.

The $n$-fold product is an $(n-1)$ ary operation on $n$ variables. In this notation two-fold products are the usual products in the ringed set $\mathscr{R}$ and the product

(2) We strongly urge the reader to follow the details by considering the case $n=4$. 
$R_{j} \times R_{k} \rightarrow R(j, k)$ is denoted by $\theta_{2}(j, k) . E_{n}\left(j_{1}, \ldots, j_{n}\right)$ is the fibre space induced from the canonical path fibrations as follows:

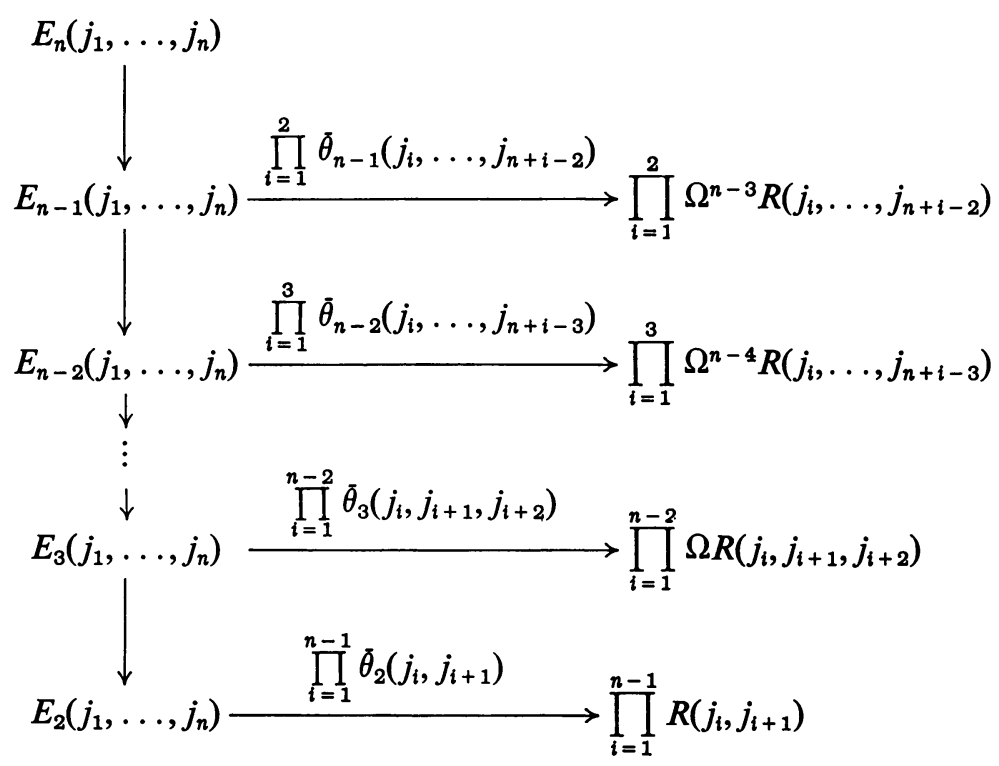

FIGURE 1

where $\bar{\theta}_{k}\left(j_{i}, \ldots, j_{i+k-1}\right)$ is the composite of a canonical projection, $E_{k}\left(j_{1}, \ldots, j_{n}\right)$ $\rightarrow E_{k}\left(j_{i}, \ldots, j_{k+i-1}\right)$ and the $k$-fold product in the universal example,

$$
E_{k}\left(j_{i}, \ldots, j_{k+i-1}\right)
$$

We now make this definition precise. Let $\mathscr{R}$ be a ringed set. For all distinguished $m$-tuples of spaces, $\left(R_{j_{1}}, \ldots, R_{j_{m}}\right), m \geqq 2$, we set $E_{2}\left(j_{1}, \ldots, j_{m}\right)=R_{j_{1}} \times \cdots \times R_{j_{m}}$ and $\theta_{2}\left(j_{1}, j_{2}\right): R_{j_{1}} \times R_{j_{2}} \rightarrow R\left(j_{1}, j_{2}\right)$ equal to the product in $\mathscr{R}$. Assume inductively that for $2 \leqq k<n$ and $m \geqq k$ we have defined $E_{k}\left(j_{1}, \ldots, j_{m}\right)$ and

such that

$$
\theta_{k}\left(j_{1}, \ldots, j_{k}\right): E_{k}\left(j_{1}, \ldots, j_{k}\right) \rightarrow \Omega^{k-2} R\left(j_{1}, \ldots, j_{k}\right)
$$

(a) if $1 \leqq s \leqq m$, and $k-1 \leqq t \leqq m-s$ there exists a projection

$$
q_{s, t}^{k}: E_{k}\left(j_{1}, \ldots, j_{m}\right) \rightarrow E_{k}\left(j_{s}, \ldots, j_{s+t}\right)
$$

such that the following diagram commutes for $s \leqq r<r+k-1 \leqq s+t$.

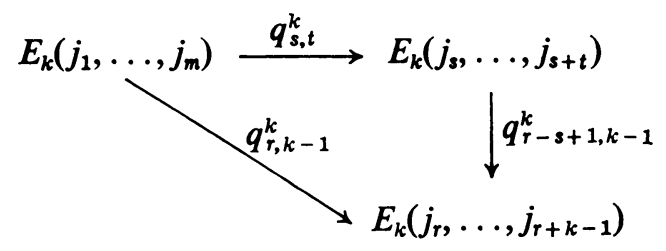


(b) $E_{k}\left(j_{1}, \ldots, j_{m}\right)$ is the fibration induced from the path fibration over $\prod_{i=1}^{m-k+2} \Omega^{k-3} R\left(j_{1}, \ldots, j_{i+k-2}\right)$ by

$\prod_{i=1}^{m-k+2} \theta_{k-1}\left(j_{i}, \ldots, j_{i+k-2}\right) q_{i, k-2}^{k-1}: E_{k-1}\left(j_{1}, \ldots, j_{m}\right) \rightarrow \prod_{i=1}^{m-k+2} \Omega^{k-3} R\left(j_{i}, \ldots, j_{i+k-2}\right)$.

(c) For $1 \leqq i \leqq k-1$ and $2 \leqq k<n$

$$
\left(\theta_{k}\left(j_{1}, \ldots, j_{k}\right)\right)^{i}=h\left(j_{1}, \ldots, j_{i}\right) \cdot h\left(j_{i+1}, \ldots, j_{k}\right)
$$

where the product and face operations are as described in $\S 1$ and for $t>0$

$$
h\left(j_{i}, \ldots, j_{i+t}\right): E_{k}\left(j_{1}, \ldots, j_{k}\right) \rightarrow P \Omega^{t-1} R\left(j_{i}, \ldots, j_{i+t}\right)
$$

is the composite of fibre space projections

$$
E_{k}\left(j_{1}, \ldots, j_{k}\right) \rightarrow E_{k-1}\left(j_{1}, \ldots, j_{k}\right) \rightarrow \cdots \rightarrow E_{t+2}\left(j_{1}, \ldots, j_{k}\right)
$$

and the canonical projection

$$
E_{t+2}\left(j_{1}, \ldots, j_{k}\right) \rightarrow P \Omega^{t-1} R\left(j_{i}, \ldots, j_{i+t}\right)
$$

(by condition (b) $E_{t+2} \subset E_{t+1} \times \Pi P \Omega^{t-1} R\left(j_{i}, \ldots, j_{i+t}\right)$ ) and $h\left(j_{i}\right): E_{k}\left(j_{1}, \ldots, j_{k}\right) \rightarrow R_{j_{t}}$ is the composite

$$
E_{k}\left(j_{1}, \ldots, j_{k}\right) \rightarrow \cdots \rightarrow E_{2}\left(j_{1}, \ldots, j_{k}\right)=R_{j_{1}} \times \cdots \times R_{j_{k}} \rightarrow R_{j_{i}} .
$$

$h\left(j_{i}, \ldots, j_{i+t}\right)$ is the canonical null-homotopy in $E_{k}\left(j_{1}, \ldots, j_{n}\right)$ of the $(t+1)$-fold product corresponding to $R_{j_{1}}, \ldots, R_{j_{i+t}}$.

Definition 2.1. For $m \geqq n$ define $E_{n}\left(j_{1}, \ldots, j_{m}\right)$ to be the fibre space induced from the canonical path fibration by

where

$$
\Phi_{m}^{n-1}: E_{n-1}\left(j_{1}, \ldots, j_{m}\right) \rightarrow \prod_{i=1}^{m-n+2} \Omega^{n-3} R\left(j_{i}, \ldots, j_{i+n-2}\right)
$$

$$
\Phi_{m}^{n-1}=\prod_{i=1}^{m-n+2} \theta_{n-1}\left(j_{i}, \ldots, j_{i+n-2}\right) q_{i, n-2}^{n-1}
$$

The composite $\theta_{n-1}\left(j_{i}, \ldots, j_{i+n-2}\right) q_{i, n-2}^{n-1}$ is the map denoted $\bar{\theta}_{n-1}\left(j_{i}, \ldots, j_{i+n-2}\right)$ in Figure 1 above.

Thus $E_{n}\left(j_{1}, \ldots, j_{m}\right)$ is the set of $(m-n+3)$-tuples $\left(x, \eta_{1}, \ldots, \eta_{m-n+2}\right)$ where $x \in E_{n-1}\left(j_{1}, \ldots, j_{m}\right)$ and $\eta_{i} \in P \Omega^{n-3} R\left(j_{i}, \ldots, j_{i+n-2}\right)$ is a null-homotopy of $\theta_{n-1}\left(j_{i}, \ldots, j_{i+n-2}\right) q_{i, n-2}^{n-1}(x)$. (We shall write $\eta_{i}(1)=\theta_{n-1}\left(j_{i}, \ldots, j_{i+n-2}\right) q_{i, n-2}^{n-1}(x)$ to denote this.)

For $0 \leqq s \leqq m$ and $n-1 \leqq t \leqq m-s$ define

by

$$
q_{s, t}^{n}: E_{n}\left(j_{1}, \ldots, j_{m}\right) \rightarrow E_{n}\left(j_{s}, \ldots, j_{s+t}\right)
$$

$$
q_{s, t}^{n}\left(x, \eta_{1}, \ldots, \eta_{m-n+2}\right)=\left(q_{s, t}^{n-1}(x), \eta_{s}, \ldots, \eta_{s+t-n+2}\right)
$$


Using the inductive hypothesis on $q_{s, t}^{n-1}$ one readily verifies that $q_{s, t}^{n}$ satisfies the properties of (a) above. This completes the definition of $E_{n}\left(j_{1}, \ldots, j_{m}\right)$.

Definition 2.2. The $n$-fold universal product corresponding to $R_{j_{1}}, \ldots, R_{j_{n}}$,

$$
\theta_{n}\left(j_{1}, \ldots, j_{n}\right): E_{n}\left(j_{1}, \ldots, j_{n}\right) \rightarrow \Omega^{n-2} R\left(j_{1}, \ldots, j_{n}\right)
$$

is defined by $\left(\theta_{n}\left(j_{1}, \ldots, j_{n}\right)\right)^{i}=h\left(j_{1}, \ldots, j_{i}\right) \cdot h\left(j_{i+1}, \ldots, j_{n}\right)$ for $i=1, \ldots, n-1$ and $\theta_{n}\left(j_{1}, \ldots, j_{n}\right) \mid L^{n}=*$.

The fact that this is well defined follows at once from condition (c). Clearly condition (c) is true for $n$. This completes the definition of the universal higher product.

Definition 2.3. Given a map $\varphi: X \rightarrow E_{n}\left(j_{1}, \ldots, j_{n}\right)$ define $M_{n}(\varphi)$, the $n$-fold Massey product (internal product), to be $(\varphi)^{*}\left[\theta_{n}\right] \in\left[X, \Omega^{n-2} R\left(j_{1}, \ldots, j_{n}\right)\right]$.

We say $\varphi: X \rightarrow E_{n}\left(j_{1}, \ldots, j_{n}\right)$ is of type $\left(f_{1}, \ldots, f_{n}\right)$ if $h\left(j_{i}\right) \varphi \sim f_{i}, i=1, \ldots, n$ or equivalently $\varphi$ is a lifting of

$$
X \stackrel{\Delta}{\longrightarrow} X^{n} \stackrel{f_{1} \times \cdots \times f_{n}}{\longrightarrow} R_{j_{1}} \times \cdots \times R_{j_{n}}
$$

where $\Delta$ is the diagonal and $f_{i}: X \rightarrow R_{j_{i}}$.

Definition 2.4. The set of $n$-fold Massey products of type $\left(f_{1}, \ldots, f_{n}\right)$ is denoted $\left\langle f_{1}, \ldots, f_{n}\right\rangle$ and defined by

$$
\left\langle f_{1}, \ldots, f_{n}\right\rangle=\left\{M_{n}(\varphi) \mid \varphi: X \rightarrow E_{n}\left(j_{1}, \ldots, j_{n}\right) \text { is of type }\left(f_{1}, \ldots, f_{n}\right)\right\} .
$$

For "nice" spaces $\Sigma\left(X_{1} \times \cdots \times X_{n}\right) \sim \bigvee \Sigma \wedge\left(X_{i_{1}}, \ldots, X_{i_{i}}\right)$ where the sum is taken over all $1 \leqq i_{1}<i_{2}<\cdots<i_{j} \leqq n$. Since

$$
\left[X_{1} \times \cdots \times X_{n}, \Omega Y\right]=\left[\Sigma\left(X_{1} \times \cdots \times X_{n}\right), Y\right]
$$

it follows that $\left[\bigwedge\left(X_{1}, \ldots, X_{n}\right), \Omega Y\right]$ is a direct summand of $\left[X_{1} \times \cdots \times X_{n}, \Omega Y\right]$. Let $q$ be the projection onto this summand.

Definition 2.5. Given $\varphi: X_{1} \times \cdots \times X_{n} \rightarrow E_{n}\left(j_{1}, \ldots, j_{n}\right)$ the $n$-fold Toda product (external product)

$$
T_{n}(\varphi) \in\left[\bigwedge\left(X_{1}, \ldots, X_{n}\right), \Omega^{n-2} R\left(j_{1}, \ldots, j_{n}\right)\right]
$$

is defined to be $q\left(\varphi^{*}\left[\theta_{n}\right]\right)$.

The definition of $\left\{f_{1}, \ldots, f_{n}\right\}$ which would be analogous to Definition 2.4 makes $\left\{f_{1}, \ldots, f_{n}\right\}$ into too large a set, i.e. the indeterminacy of the construction is too great. Thus we consider only those maps $\varphi: X_{1} \times \cdots \times X_{n} \rightarrow E_{n}\left(j_{1}, \ldots, j_{n}\right)$ which have the property that for each $k$-tuple $(i, \ldots, i+k-1)$ with $2 \leqq k<n$ and $1 \leqq i$ $\leqq n-k+1$ there is a map

$$
\varphi(i, \ldots, i+k-1): X_{i} \times \cdots \times X_{i+k-1} \rightarrow E_{k+1}\left(j_{1}, \ldots, j_{n}\right)
$$


such that the following diagram commutes.

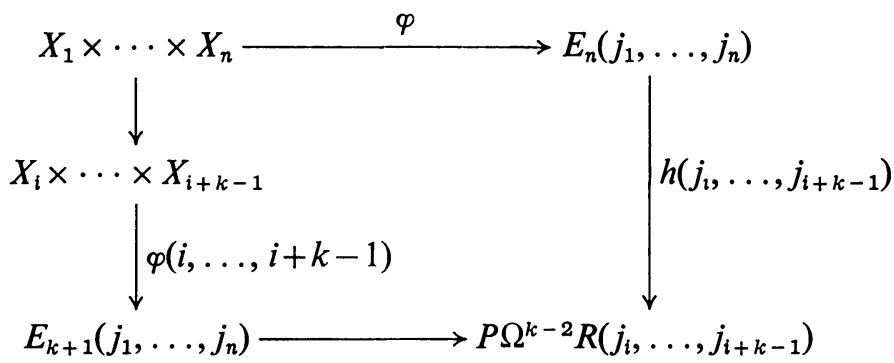

where the unlabeled arrows are canonical projections. We call such maps special.

A map $\varphi: X_{1} \times \cdots \times X_{n} \rightarrow E_{n}\left(j_{1}, \ldots, j_{n}\right)$ is said to be of type $\left(f_{1}, \ldots, f_{n}\right)$ if $\varphi$ is a lifting of

$$
f_{1} \times \cdots \times f_{n}: X_{1} \times \cdots \times X_{n} \rightarrow R_{j_{1}} \times \cdots \times R_{j_{n}} .
$$

Definition 2.6. The set of $n$-fold Toda products of type $\left(f_{1}, \ldots, f_{n}\right)$ is denoted $\left\{f_{1}, \ldots, f_{n}\right\}$ and is defined by

$\left\{f_{1}, \ldots, f_{n}\right\}=\left\{T_{n}(\varphi) \mid \varphi: X_{1} \times \cdots \times X_{n} \rightarrow E_{n}\left(j_{1}, \ldots, j_{n}\right)\right.$ is special of type $\left.\left(f_{1}, \ldots, f_{n}\right)\right\}$.

All of the properties of $\left\{f_{1}, \ldots, f_{n}\right\}$ except Theorem 3.9 are true whether or not special maps are used.

If the external product is defined so is the internal and we have:

Proposition 2.7. $\Delta^{*}\left\{f_{1}, \ldots, f_{n}\right\} \subset\left\langle f_{1}, \ldots, f_{n}\right\rangle$ where $\Delta: X \rightarrow X^{n}$ is the diagonal map.

The Massey product may, however, be defined without the Toda product being defined. Such, for example, is the case in singular cohomology.

Finally we remark that for $n>2,\left\{f_{1}, \ldots, f_{n}\right\}$ and $\left\langle f_{1}, \ldots, f_{n}\right\rangle$ may be the empty set.

3. Properties of the higher products. In this section we study the properties of the products defined in $\S 2$. We note that $M_{n}(\varphi)$ and $T_{n}(\varphi)$ are well defined elements of the appropriate homotopy groups while $\left\langle f_{1}, \ldots, f_{n}\right\rangle$ and $\left\{f_{1}, \ldots, f_{n}\right\}$ are subsets of these groups. Thus for properties of $M_{n}(\varphi)$ and $T_{n}(\varphi)$ in which we use "=" we must in general use " $\subset$ " for $\left\langle f_{1}, \ldots, f_{n}\right\rangle$ and $\left\{f_{1}, \ldots, f_{n}\right\}$.

TheOREM 3.1 (NATURALITY). Let $f: X \rightarrow Y$ and

(a) let $\varphi: Y \rightarrow E_{n}\left(j_{1}, \ldots, j_{n}\right)$ then $f^{*} M_{n}(\varphi)=M_{n}(\varphi f)$.

(b) Let $y_{i}: Y \rightarrow R_{y_{i}}, 1 \leqq i \leqq n$ then $f^{*}\left\langle y_{1}, \ldots, y_{n}\right\rangle \subset\left\langle y_{1} f, \ldots, y_{n} f\right\rangle$.

Let $f_{i}: X_{i} \rightarrow Y_{i}, 1 \leqq i \leqq n$ and

(c) let $\varphi: Y_{1} \times \cdots \times Y_{n} \rightarrow E_{n}\left(j_{1}, \ldots, j_{n}\right)$ then

$$
\left(\bigwedge\left(f_{1}, \ldots, f_{n}\right)\right)^{*} T_{n}(\varphi)=T_{n}\left(\varphi\left(f_{1} \times \cdots \times f_{n}\right)\right)
$$


(d) let $y_{i}: Y_{i} \rightarrow R_{j_{i}}, 1 \leqq i \leqq n$ then

$$
\left(\bigwedge\left(f_{1}, \ldots, f_{n}\right)\right)^{*}\left\{y_{1}, \ldots, y_{n}\right\} \subset\left\{y_{1} f_{1}, \ldots, y_{n} f_{n}\right\}
$$

Proof. Clearly (b) follows from (a) and (d) follows from (c). To prove (a) we have

$$
f^{*} M_{n}(\varphi)=f^{*} \varphi^{*}\left[\theta_{n}\right]=(\varphi f)^{*}\left[\theta_{n}\right]=M_{n}(\varphi f) .
$$

To prove (c) we note that the following diagram commutes.

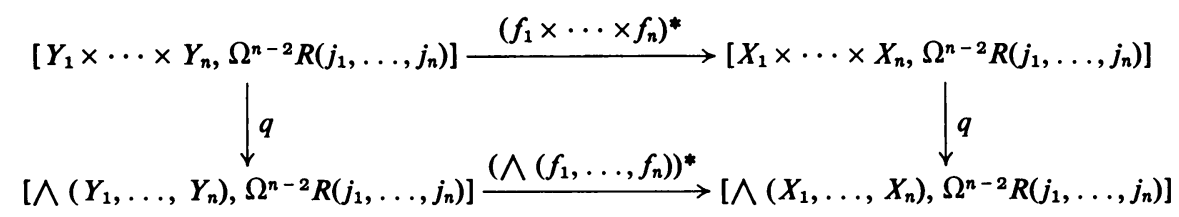

The proof of (c) is then immediate.

Let $\mathscr{R}$ and $\mathscr{S}$ be ringed sets. $F: \mathscr{R} \rightarrow \mathscr{S}$ is said to be a morphism of ringed sets if $F=\left\{f(j): R_{j} \rightarrow S_{j}\right.$ for $\left.j \in J\right\}$ such that the following diagram commutes for all distinguished $(i, j) \in J \times J$.

$$
\begin{aligned}
& R_{i} \times R_{j} \stackrel{f(i) \times f(j)}{\longrightarrow} S_{i} \times S_{j} \\
& \quad \theta_{2}^{R}(i, j) \\
& R(i, j) \stackrel{f(i, j)}{\downarrow} S(i, j)
\end{aligned}
$$

THEOREM 3.2. Let $F: \mathscr{R} \rightarrow \mathscr{S}$ be a morphism of ringed sets and let $\theta_{n}^{R}$ and $\theta_{n}^{S}$ be the $n$-fold universal products corresponding to $R_{j_{1}}, \ldots, R_{j_{n}}$ and $S_{j_{1}}, \ldots, S_{j_{n}}$ respectively. Then there exists $F_{n}: E_{n}^{R}\left(j_{1}, \ldots, j_{n}\right) \rightarrow E_{n}^{S}\left(j_{1}, \ldots, j_{n}\right)$ which covers $f_{j_{1}} \times \cdots \times f_{j_{n}}$ such that $\theta_{n}^{S} F_{n}=\Omega^{n-2}\left(f\left(j_{1}, \ldots, j_{n}\right)\right) \theta_{n}^{R}$.

Proof. We shall define $g_{n, k}: E_{k}^{R}\left(j_{1}, \ldots, j_{n}\right) \rightarrow E_{k}^{S}\left(j_{1}, \ldots, j_{n}\right), 2 \leqq k \leqq n$, such that the following diagram commutes.

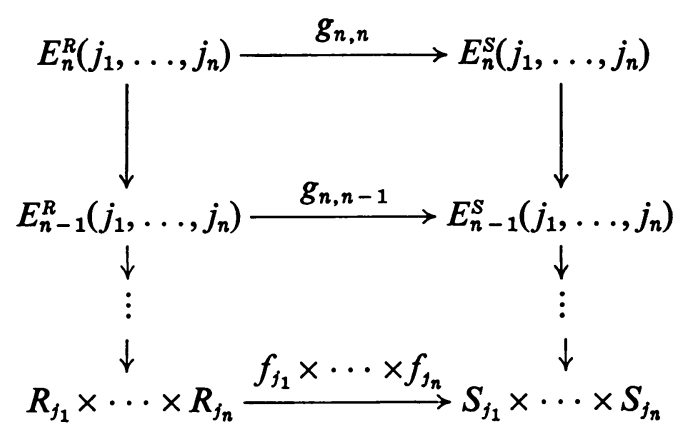

We then set $g_{n, n}=F_{n}$. If $n=2(3 \mathrm{~b})$ holds trivially and the theorem is simply the definition of a morphism of ringed sets. Assume inductively that $g_{m, k}$ is defined for $m<n$ such that $F_{m}=g_{m, m}$ satisfies the theorem. $g_{n, 2}$ is defined to be $f_{j_{1}} \times \cdots \times f_{j_{n}}$. 
Assume inductively that $g_{n, i}$ is defined for $2 \leqq i<k$ and satisfies $q_{s, t}^{i} g_{n, i}=g_{t+1, i} q_{s, t}^{i}$ where $1 \leqq s \leqq n$ and $i-1 \leqq t \leqq n-s$ and $i<k$.

Denote the maps, constructions, etc. related to $\mathscr{R}$ by using super $R$ and those related to $\mathscr{S}$ by using super $S$.

Recall that

$$
\begin{array}{r}
E_{k}^{R}\left(j_{1}, \ldots, j_{n}\right)=\left\{\left(x, \eta_{1}, \ldots, \eta_{n-k+2}\right) \in E_{k-1}^{R} \times \prod_{i=1}^{n-k+2} P \Omega^{k-3} R\left(j_{i}, \ldots, j_{i+k-2}\right)\right. \\
\text { such that } \left.\eta_{i}(1)=\theta_{k-1}^{R}\left(j_{i}, \ldots, j_{i+k-2}\right) q_{i, k-2}^{k-1}(x)\right\} .
\end{array}
$$

Define $g_{n, k}$ by

$$
g_{n, k}\left(x, \eta_{1}, \ldots, \eta_{n-k+2}\right)=\left(g_{n, k-1}(x), \bar{\eta}_{1}, \ldots, \bar{\eta}_{n-k+2}\right)
$$

where $\bar{\eta}_{\imath}=P \Omega^{k-3}\left(f\left(j_{i}, \ldots, j_{i+k-2}\right)\right)\left(\eta_{i}\right)$.

$$
\begin{aligned}
\bar{\eta}_{i}(1) & =\Omega^{k-3}\left(f\left(j_{i}, \ldots, j_{i+k-2}\right)\right) \theta_{k-1}^{R}\left(j_{i}, \ldots, j_{i+k-2}\right) q_{i, k-2}^{k-1}(x) \\
& =\theta_{k-1}^{S}\left(j_{i}, \ldots, j_{i+k-2}\right) g_{k-1, k-1} q_{i, k-2}^{k-1}(x) \\
& =\theta_{k-1}^{S}\left(j_{i}, \ldots, j_{i+k-2}\right) q_{i, k-2}^{k-1} g_{n, k-1}(x)
\end{aligned}
$$

by the inductive hypothesis. Thus

$$
g_{n, k}: E_{k}^{R}\left(j_{1}, \ldots, j_{n}\right) \rightarrow E_{k}^{S}\left(j_{1}, \ldots, j_{n}\right) .
$$

This definition satisfies ( $3 b$ ) and the commutation formula with $q_{s, t}^{k}$. We must still show that $g_{n, n}=F_{n}$ satisfies the theorem. Since $F$ is a morphism of ringed sets, for each $t, 1 \leqq t<n$ we have

$$
\begin{aligned}
\left(\Omega^{n-2} f\left(j_{1}, \ldots, j_{n}\right) \theta_{n}^{R}\right)^{t} & =P \Omega^{n-3}\left(f\left(j_{1}, \ldots, j_{n}\right)\right)\left(h^{R}\left(j_{1}, \ldots, j_{t}\right) \cdot h^{R}\left(j_{t+1}, \ldots, j_{n}\right)\right) \\
& =\left(P \Omega^{t-2}\left(f\left(j_{1}, \ldots, j_{t}\right) h^{R}\left(j_{1}, \ldots, j_{t}\right)\right)\right. \\
& =\left(\theta_{n}^{S} g_{n, n}\right)^{t} .
\end{aligned}
$$

Thus the theorem is proven.

COROLlaRY 3.3. Let $F: \mathscr{R} \rightarrow \mathscr{S}$ be a morphism of ringed sets

$$
\begin{aligned}
\left(\Omega^{n-2} f\left(j_{1}, \ldots, j_{n}\right)\right)_{*}\left\{x_{1}, \ldots, x_{n}\right\} & \subset\left\{f_{j_{1}} x_{1}, \ldots, f_{j_{n}} x_{n}\right\} \\
\left(\Omega^{n-2} f\left(j_{1}, \ldots, j_{n}\right)\right)_{*}\left\langle x_{1}, \ldots, x_{n}\right\rangle & \subset\left\langle f_{j_{1}} x_{1}, \ldots, f_{j_{n}} x_{n}\right\rangle
\end{aligned}
$$

where $x_{i}: X_{i} \rightarrow R_{i}$ in (a) and $x_{i}: X \rightarrow R_{i}$ in (b).

REMARK. Homotopy commutativity of (3a) is not enough to ensure the existence of $F_{n}$. We may define higher degrees of homotopy morphisms for $F$ which ensure that Theorem 3.2 holds. Alternately we say that $F$ is a homotopy morphism of ringed sets of order $n$ if there exists $F_{n}$ as above such that

$$
\theta_{n}^{R} F_{n} \sim \Omega^{n-2} f\left(j_{1}, \ldots, j_{n}\right) \theta_{n}^{S}
$$


If $\left[R_{i}, R_{i}\right]$ is a group for each $R_{i} \in \mathscr{R}$ we define $k: R_{i} \rightarrow R_{i}$ to be $(\mathrm{Id}+\cdots+\mathrm{Id})$ ( $k$ times). We say $\mathscr{R}$ is linear if the following diagram commutes for distinguished $(i, j)$.

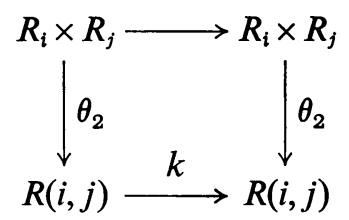

where the top map is either $1 \times k$ or $k \times 1$.

COROLlaRy 3.4 (LINEARITY). If $\mathscr{R}$ is linear then for each $t, 1 \leqq t \leqq n$, there is a map $k_{t}^{n}: E_{n}\left(j_{1}, \ldots, j_{n}\right) \rightarrow E_{n}\left(j_{1}, \ldots, j_{n}\right)$ which covers

$$
1 \times \cdots \times k \times \cdots \times 1: R_{j_{1}} \times \cdots \times R_{j_{t}} \times \cdots \times R_{j_{n}} \rightarrow R_{j_{1}} \times \cdots \times R_{j_{t}} \times \cdots \times R_{j_{n}}
$$

and such that $\theta_{n} k_{t}^{n}=k \theta_{n}$.

Proof. By enlarging the ringed set $\mathscr{R}$ to include many (differently named) copies of each $R_{i} \in \mathscr{R}$ we may assume without loss of generality that $\left(R_{i}, R_{i}\right)$ is never distinguished. (It may of course be the case that for $i \neq j, R_{i}=R_{j}$ and $\left(R_{i}, R_{j}\right)$ is distinguished.)

Define $k_{t}\left(j_{1}, \ldots, j_{n}\right): R\left(j_{1}, \ldots, j_{n}\right) \rightarrow R\left(j_{1}, \ldots, j_{n}\right)$ by

$$
\begin{aligned}
k_{t}\left(j_{1}, \ldots, j_{n}\right) & =k & & \text { if } j_{s}=j_{t} \text { for some } s \\
& =1 & & \text { otherwise. }
\end{aligned}
$$

Under the above restriction it is easily seen that $\mathscr{R}$ is linear if and only if $K_{t}$ $=\left\{k_{t}\left(j_{1}, \ldots, j_{n}\right)\right\}: \mathscr{R} \rightarrow \mathscr{R}$ is a morphism of ringed sets. The corollary then follows from Theorem 3.2 by noting that $\Omega k$ is again $k$ times the appropriate identity.

COROllary 3.5. If $\mathscr{R}$ is linear

(a) let $\varphi: X_{1} \times \cdots \times X_{n} \rightarrow E_{n}\left(j_{1}, \ldots, j_{n}\right)$ be of type $\left(f_{1}, \ldots, f_{n}\right)$, then $\left(k_{j}^{n}\right) \varphi$ is of type $\left(f_{1}, \ldots, k f_{j}, \ldots, f_{n}\right)$ and $T_{n}\left(k_{j}^{n} \varphi\right)=k T_{n}(\varphi)$;

(b) $\left\{f_{1}, \ldots, k f_{j}, \ldots, f_{n}\right\} \supset k\left\{f_{1}, \ldots, f_{n}\right\}$;

(c) let $\varphi: X \rightarrow E_{n}\left(j_{1}, \ldots, j_{n}\right)$ be of type $\left(f_{1}, \ldots, f_{n}\right)$ then $\left(k_{j}^{n}\right) \varphi$ is of type $\left(f_{1}, \ldots, k f_{j}, \ldots, f_{n}\right)$ and $M_{n}\left(k_{j}^{n} \varphi\right)=k M_{n}(\varphi)$;

(d) $\left\langle f_{1}, \ldots, k f_{j}, \ldots, f_{n}\right\rangle \supset k\left\langle f_{1}, \ldots, f_{n}\right\rangle$.

If $X_{i}$ is an $H^{\prime}$-space (e.g. suspension) then $k f_{i}$ is defined for $f_{i}: X_{i} \rightarrow R_{j_{i}}$ and we may once again discuss linearity. Here the situation is easier and no hypothesis need be put on $\mathscr{R}$. Since $X_{i}$ is an $H^{\prime}$-space, $\bigwedge\left(X_{1}, \ldots, X_{i}, \ldots, X_{n}\right)$ is also an $H^{\prime}$ space under $\wedge(1, \ldots, w, \ldots, 1)$ where $w$ is the comultiplication in $X_{i}$. Thus $\bigwedge(1, \ldots, k, \ldots, 1)$ is $k$ times the identity in $\wedge\left(X_{1}, \ldots, X_{n}\right)$. The following is then a consequence of $3.1(c)$. 
THEOREM 3.6. If $\varphi: X_{1} \times \cdots \times X_{n} \rightarrow E_{n}\left(j_{1}, \ldots, j_{n}\right)$ is of type $\left(f_{1}, \ldots, f_{n}\right)$ then $\varphi(1 \times \cdots \times k \times \cdots \times 1)$ is of type $\left(f_{1}, \ldots, k f_{i}, \ldots, f_{n}\right)$ and

(a) $T_{n}(\varphi(1 \times \cdots \times k \times \cdots \times 1))=k T_{n}(\varphi)$,

(b) $k\left\{f_{1}, \ldots, f_{n}\right\} \subset\left\{f_{1}, \ldots, k f_{i}, \ldots, f_{n}\right\}$.

(We omit the corresponding results on Massey products since we show below that all Massey products vanish on suspensions.)

Theorem 3.7 (Associativity). In $\left[E_{n-1}\left(j_{1}, \ldots, j_{n}\right), \Omega^{n-3} R\left(j_{1}, \ldots, j_{n}\right)\right]$

$$
\theta_{n-1}\left(j_{1}, \ldots, j_{n-1}\right) \cdot h\left(j_{n}\right)=(-1)^{n+1} h\left(j_{1}\right) \cdot \theta_{n-1}\left(j_{2}, \ldots, j_{n}\right) .
$$

Proof. In the notation of $\$ 2$ consider

$$
\sum_{t=2}^{n-2}(-1)^{t+1} h\left(j_{1}, \ldots, j_{t}\right) \cdot h\left(j_{t+1}, \ldots, j_{n}\right) .
$$

This is a map from $E_{n-1}\left(j_{1}, \ldots, j_{n}\right)$ to

$$
\left\{\left(I^{n-1}-1 \text { st and }(n-1) \text { st top faces), } R\left(j_{1}, \ldots, j_{n}\right)\right\}\right. \text {. }
$$

This can be considered as a map $E_{n-1}\left(j_{1}, \ldots, j_{n}\right) \rightarrow\left(\Omega^{n-3} R\left(j_{1}, \ldots, j_{n}\right)\right)^{I}$ which is the desired homotopy.

CoRollary 3.8. If $\varphi: X \rightarrow E_{n-1}\left(j_{1}, \ldots, j_{n}\right)$ is of type $\left(f_{1}, \ldots, f_{n}\right)$ then

(a) $f_{1} \cdot M_{n-1}\left(q_{2, n-2}^{n-1} \varphi\right)=(-1)^{n+1} M_{n-1}\left(q_{1, n-2}^{n-1} \varphi\right) \cdot f_{n}$,

(b) $f_{1} \cdot\left\langle f_{2}, \ldots, f_{n}\right\rangle \cap(-1)^{n+1}\left\langle f_{1}, \ldots, f_{n-1}\right\rangle \cdot f_{n}$ is nonempty.

Let $\varphi: X_{1} \times \cdots \times X_{n} \rightarrow E_{n-1}\left(j_{1}, \ldots, j_{n}\right)$ be of type $\left(f_{1}, \ldots, f_{n}\right)$ then

(c) the projections of $f_{1} \cdot T_{n-1}\left(q_{2, n-2}^{n-1} \varphi\right)$ and $(-1)^{n+1} T_{n-1}\left(q_{1, n-2}^{n-2} \varphi\right) \cdot f_{n}$ into [ $\left.\wedge\left(X_{1}, \ldots, X_{n}\right), \Omega^{n-3} R\left(j_{1}, \ldots, j_{n}\right)\right]$ are equal.

(d) $f_{1} \cdot\left\{f_{2}, \ldots, f_{n}\right\} \cap(-1)^{n+1}\left\{f_{1}, \ldots, f_{n-1}\right\} \cdot f_{n}$ is nonempty.

Proof. To see that (c) holds, one observes that the following diagram commutes. All maps are projections into direct summands.

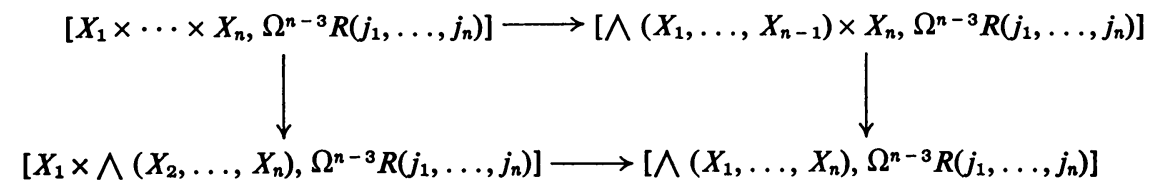

The proofs are then all evident.

Hardie [2] has introduced the notion of a derived homotopy operation. Given a relation involving an $(n-1)$ ary homotopy operation, one obtains a derived nary operation. In this language the secondary product is the operation derived from the associativity relation. Theorem 3.7 gives a higher associativity relation involving the $(n-2)$ ary products. The $(n-1)$ ary product $\theta_{n}$ is then seen to be derived from higher associativity. This relation enables us to calculate the "modulus" of the construction at the last step. 
THeOREM 3.9. (a) Let $\varphi_{n-1}: X \rightarrow E_{n-1}\left(j_{1}, \ldots, j_{n}\right)$ be a map of type $\left(f_{1}, \ldots, f_{n}\right)$ which can be lifted to $E_{n}\left(j_{1}, \ldots, j_{n}\right)$ then $\left\{M_{n}(\varphi) \mid \varphi\right.$ lifts $\left.\varphi_{n-1}\right\}$ is a coset of

$$
f_{1} \cdot\left[X, \Omega^{n-2} R\left(j_{2}, \ldots, j_{n}\right)\right]+\left[X, \Omega^{n-2} R\left(j_{1}, \ldots, j_{n-1}\right)\right] \cdot f_{n} .
$$

(b) Let $\varphi_{n-1}: X_{1} \times \cdots \times X_{n} \rightarrow E_{n-1}\left(j_{1}, \ldots, j_{n}\right)$ be a special map of type $\left(f_{1}, \ldots, f_{n}\right)$ which can be lifted to $E_{n}\left(j_{1}, \ldots, j_{n}\right)$ then $\left\{T_{n}(\varphi) \mid \varphi\right.$ lifts $\left.\varphi_{n-1}\right\}$ is a coset of $f_{1} \cdot\left[\bigwedge\left(X_{2}, \ldots, X_{n}\right), \Omega^{n-2} R\left(j_{2}, \ldots, j_{n}\right)\right]+\left[\bigwedge\left(X_{1}, \ldots, X_{n-1}\right), \Omega^{n-2} R\left(j_{1}, \ldots, j_{n-1}\right)\right] \cdot f_{n}$ (where these products are projected into $\left.\left[\bigwedge\left(X_{1}, \ldots, X_{n}\right), \Omega^{n-2} R\left(j_{1}, \ldots, j_{n}\right)\right]\right)$.

Proof. (a) The lifting of $\varphi_{n-1}$ to $E_{n}\left(j_{1}, \ldots, j_{n}\right)$ is given by choosing null-homotopies of $M_{n-1}\left(q_{2, n-2}^{n-1} \varphi_{n-1}\right)$ and $M_{n-1}\left(q_{1, n-2}^{n-1} \varphi_{n-1}\right)$. Any two null-homotopies of $M_{n-1}\left(q_{2, n-2}^{n-1} \varphi_{n-1}\right)$ differ by an element of $\left[X, \Omega^{n-2} R\left(j_{2}, \ldots, j_{n}\right)\right]$ and each element of this group can be expressed as the difference of two null-homotopies of $M_{n-1}\left(q_{2, n-2}^{n-1} \varphi_{n-1}\right)$. Similarly two null-homotopies of $M_{n-1}\left(q_{1, n-2}^{n-1} \varphi_{n-1}\right)$ differ by an element of $\left[X, \Omega^{n-2} R\left(j_{1}, \ldots, j_{n-1}\right)\right]$. Thus if $\varphi$ and $\varphi^{\prime}$ are liftings of $\varphi_{n-1}$

$$
\left(\varphi^{*}\left[\theta_{n}\right]-\left(\varphi^{\prime}\right)^{*}\left[\theta_{n}\right]\right) \in f_{1} \cdot\left[X, \Omega^{n-2} R\left(j_{2}, \ldots, j_{n}\right)\right]+\left[X, \Omega^{n-2} R\left(j_{1}, \ldots, j_{n-1}\right)\right] \cdot f_{n} .
$$

(b) Because $\varphi$ is special, the null-homotopies of

$$
T_{n-1}\left(q_{2, n-2}^{n-1} \varphi_{n-1}\right) \text { and } T_{n-1}\left(q_{1, n-2}^{n-1} \varphi_{n-1}\right)
$$

are determined up to elements of

$$
\left[X_{2} \times \cdots \times X_{n}, \Omega^{n-2} R\left(j_{2}, \ldots, j_{n}\right)\right] \quad \text { and }\left[X_{1} \times \cdots \times X_{n-1}, \Omega^{n-2} R\left(j_{1}, \ldots, j_{n-1}\right)\right]
$$

respectively and the theorem follows as above.

If $\varphi$ were not special the homotopies in (b) would vary over

$$
\left[X_{1} \times \cdots \times X_{n}, \Omega^{n-2} R\left(j_{2}, \ldots, j_{n}\right)\right] \text { and }\left[X_{1} \times \cdots \times X_{n}, \Omega^{n-2} R\left(j_{1}, \ldots, j_{n-1}\right)\right]
$$

respectively and the cosets would be larger than above. This is the only property of the higher Toda products which uses "specialness".

One notes that the more general problem of determining the "modulus" of $\left\langle f_{1}, \ldots, f_{n}\right\rangle$ or $\left\{f_{1}, \ldots, f_{n}\right\}$ is much more difficult.

THEOREM 3.10. Let $\varphi: \Sigma X \rightarrow E_{n}\left(j_{1}, \ldots, j_{n}\right)$ then $M_{n}(\varphi)=0$.

Proof. We prove there exists $\varphi^{\prime} \sim \varphi$ such that $\theta_{n}\left(j_{1}, \ldots, j_{n}\right) \varphi^{\prime}=*$. Let

$$
\varphi_{r}: \Sigma X \rightarrow E_{r}\left(j_{1}, \ldots, j_{n}\right)
$$

be such that $\varphi_{r}$ lifts $\varphi_{r-1}$ and $\varphi_{n}=\varphi$. If $r=2, \varphi_{2} \sim\left(f_{1} \times \cdots \times f_{n}\right) \Delta^{n}$ for some maps $f_{1}, \ldots, f_{n}$, where $\Delta^{n}$ is the diagonal. Since $\Sigma X$ is a suspension $\Delta^{n}$ can be factored through $\Sigma X \vee \cdots \vee \Sigma X$ (up to homotopy). Hence $\varphi_{2}$ is homotopic to the composite

$$
\Sigma X \rightarrow \Sigma X \vee \cdots \vee \Sigma X \stackrel{f_{1}|\cdots| f_{n}}{\longrightarrow} R_{j_{1}} \times \cdots \times R_{j_{n}}
$$


Call this $\varphi_{2}^{(2)}$. Clearly $\theta_{2}\left(j_{i}, j_{i+1}\right) q_{i, 1} \varphi_{2}^{(2)}=*$ for $1 \leqq i \leqq n-1$. By the homotopy lifting property there exist $\varphi_{k}^{(2)}: \Sigma X \rightarrow E_{k}\left(j_{1}, \ldots, j_{n}\right), 2 \leqq k \leqq n$, such that $\varphi_{k}^{(2)}$ lifts $\varphi_{k-1}^{(2)}$ and $\varphi_{k}^{(2)} \sim \varphi_{k}$.

Since $\theta_{2}\left(j_{i}, j_{i+1}\right) q_{i, 1}^{2} \varphi_{2}^{(2)}=*, \varphi_{3}^{(2)}$ is seen to be a map

$$
\Sigma X \rightarrow E_{2}\left(j_{1}, \ldots, j_{n}\right) \times \prod_{i=1}^{n-1} \Omega R\left(j_{i}, j_{i+1}\right) \subset E_{3}\left(j_{1}, \ldots, j_{n}\right)
$$

and hence is homotopic to a composite

$$
\Sigma X \rightarrow \Sigma X \vee \cdots \vee \Sigma X \rightarrow E_{2}\left(j_{1}, \ldots, j_{n}\right) \times \prod_{i=1}^{n-1} \Omega R\left(j_{i}, j_{i+1}\right)
$$

where the second map is $\varphi_{2}^{(2)}\left|\eta_{1}\right| \cdots \mid \eta_{n-1}$ with $\eta_{i}: \Sigma X \rightarrow \Omega R\left(j_{i}, j_{i+1}\right)$. Call this $\operatorname{map} \varphi_{3}^{(3)}$.

$\theta_{3}\left(j_{i}, j_{i+1}, j_{i+2}\right) q_{i, 2}^{3} \varphi_{3}^{(3)}$ is the "sum" of two products. Each of these products has as its factors two maps from $\Sigma X$ which have the property that at each point of $\Sigma X$ the image of at most one of them is different from $*$. Thus each product equals * and $\theta_{3}\left(j_{i}, j_{i+1}, j_{i+2}\right) q_{i, 2}^{3} \varphi_{3}^{(3)}=*$ for $1 \leqq i \leqq n-2$. By the homotopy lifting property there are maps $\varphi_{k}^{(3)}: \Sigma X \rightarrow E_{k}\left(j_{1}, \ldots, j_{n}\right)$ for $k<3$ such that $\varphi_{k}^{(3)}$ covers $\varphi_{k-1}^{(3)}$ and $\varphi_{k}^{(3)} \sim \varphi_{k}$.

We continue in this manner until we define $\varphi_{n}^{(n)}=\varphi^{\prime}$. By construction

$$
\theta_{n}\left(j_{1}, \ldots, j_{n}\right) \varphi^{\prime}=* .
$$

COROllaRY 3.11. Let $f_{i}: \Sigma X \rightarrow R_{i}, i=1, \ldots, n,\left\langle f_{1}, \ldots, f_{n}\right\rangle$ is the set consisting of the zero element.

COROllary 3.12. Let

$$
\Omega_{*}:\left[X, \Omega^{n-2} R\left(j_{1}, \ldots, j_{n}\right)\right] \rightarrow\left[\Omega X, \Omega^{n-1} R\left(j_{1}, \ldots, j_{n}\right)\right]
$$

be the loop map and let $\varphi: X \rightarrow E_{n}\left(j_{1}, \ldots, j_{n}\right)$ then $\Omega_{*} M_{n}(\varphi)=0$.

Proof. Let $\lambda: \Sigma \Omega X \rightarrow X$ be the adjoint of the identity on $\Omega X$. Then $\lambda^{*} M_{n}(\varphi)$ $=M_{n}(\varphi)=0$ by $3.1(\mathrm{a})$ and 3.11. Since $\Omega_{*}$ is the composite of $\lambda$ with the isomorphism

$$
\left[\Sigma \Omega X, \Omega^{n-2} R\left(j_{1}, \ldots, j_{n}\right)\right] \approx\left[\Omega X, \Omega^{n-1} R\left(j_{1}, \ldots, j_{n}\right)\right]
$$

the corollary follows.

COROllaRY 3.13. $\Omega E_{k}\left(j_{1}, \ldots, j_{n}\right) \sim \Pi \Omega^{t+1} R\left(j_{i}, \ldots, j_{i+t}\right)$ where the product is taken over $0 \leqq t \leqq k-2$ and $1 \leqq i \leqq n-t$.

We note that since the projection

$$
\left[X_{1} \times \cdots \times X_{n}, \Omega^{n-2} R\left(j_{1}, \ldots, j_{n}\right)\right] \rightarrow\left[\bigwedge\left(X_{1}, \ldots, X_{n}\right), \Omega^{n-2} R\left(j_{1}, \ldots, j_{n}\right)\right]
$$


is not induced by a map of topological spaces there does not exist a functorial map to make the following diagram commute.

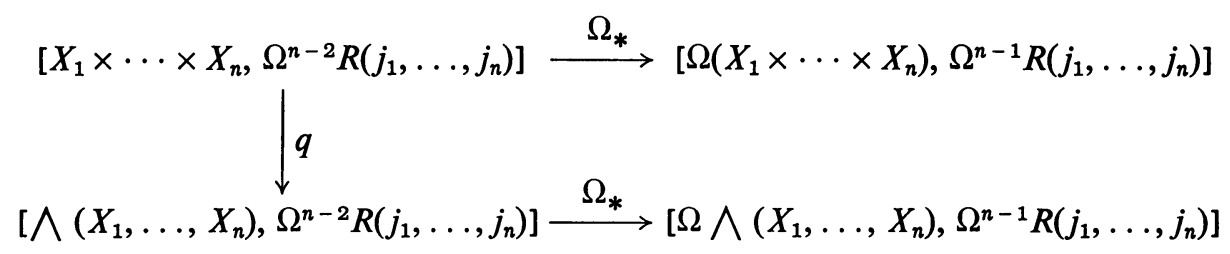

Thus although $\Omega_{*} \theta_{n}=0$ it is not true, in general, that $\Omega_{*} T_{n}(\varphi)=0$ for

$$
\varphi: X_{1} \times \cdots \times X_{n} \rightarrow E_{n}\left(j_{1}, \ldots, j_{n}\right) .
$$

The essential point in the proof of 3.10 was that $\varphi_{2}$ was defined using the diagonal in $\Sigma X$ and this can be factored through the one point union. Since this is not true for $T_{n}$, it is not true that $T_{n}$ vanishes in suspensions. On the contrary $T_{n}$ is of great interest on spheres where it represents the stable Toda bracket.

We now study the stability properties of higher products. In what follows we consider the suspension homomorphism,

to be induced by

$$
\Sigma_{*}: \pi_{n}\left(\Omega^{j} X\right) \rightarrow \pi_{n+1}\left(\Omega^{j} \Sigma X\right)
$$

$$
\Sigma(f)(t, s)(\lambda)=(t, f(s)(\lambda))
$$

where $t \in I, s \in S^{n}, \lambda \in I^{j}$. This is the composite

$$
\pi_{n}\left(\Omega^{j} X\right) \approx \pi_{n+j}(X) \rightarrow \pi_{n+j+1}(\Sigma X) \approx \pi_{n+1}\left(\Omega^{j} \Sigma X\right) .
$$

Let $\mathscr{R}$ be a ringed set and $\Sigma \mathscr{R}$ the associated ringed set defined in Example 6 of $\S 1$.

Let $E_{r}\left(j_{1}, \ldots, j_{n}\right), \theta_{n}\left(j_{1}, \ldots, j_{n}\right)$ and $E_{r}\left(\Sigma_{j_{1}}, \ldots, \Sigma_{j_{n}}\right), \theta_{n}\left(\Sigma_{j_{1}}, \ldots, \Sigma_{j_{n}}\right)$ be the universal examples and maps associated with $R_{j_{1}}, \ldots, R_{j_{n}}$ and $\Sigma R_{j_{1}}, \ldots, \Sigma R_{j_{n}}$ respectively.

THeOREM 3.14. Let $\varphi: X_{1} \times \cdots \times X_{n} \rightarrow E_{n}\left(j_{1}, \ldots, j_{n}\right)$ a map of type $\left(f_{1}, \ldots, f_{n}\right)$. There exists a map

$$
\sigma(n, n):\left\{X_{1} \times \cdots \times X_{n}, E_{n}\left(j_{1}, \ldots, j_{n}\right)\right\} \rightarrow\left\{\Sigma X_{1} \times \cdots \times \Sigma X_{n}, E_{n}\left(\Sigma_{j_{1}}, \ldots, \Sigma_{j_{n}}\right)\right\}
$$

such that $\sigma(n, n)(\varphi)$ is of type $\left(\Sigma f_{1}, \ldots, \Sigma f_{n}\right)$ and $T_{n}(\sigma(n, n)(\varphi))=\Sigma_{*}^{n} T_{n}(\varphi)$.

Proof. For each $r, k$, and $i, 2 \leqq r \leqq k$ and $1 \leqq i \leqq k-r-1$ define

$$
\begin{aligned}
\tau(r, k, i):\left\{X_{1} \times \cdots \times X_{k}, \Omega^{r-2} R\left(j_{i},\right.\right. & \left.\left., ., j_{i+r-1}\right)\right\} \\
& \rightarrow\left\{\Sigma X_{1} \times \cdots \times \Sigma X_{k}, \Omega^{r-2} \Sigma^{r} R\left(j_{i}, \ldots, j_{i+r-1}\right)\right\}
\end{aligned}
$$

by

$$
(\tau(r, k, i)(\psi))\left(t_{1}, x_{1}, t_{2}, x_{2}, \ldots, t_{k}, x_{k}\right)(\lambda)=\left(t_{i}, \ldots, t_{i+r-1}, \psi\left(x_{1}, \ldots, x_{k}\right)(\lambda)\right)
$$


where $t_{i} \in I, x_{i} \in X$ and $\lambda \in I^{r-2}$. A direct calculation shows that the following diagram commutes.

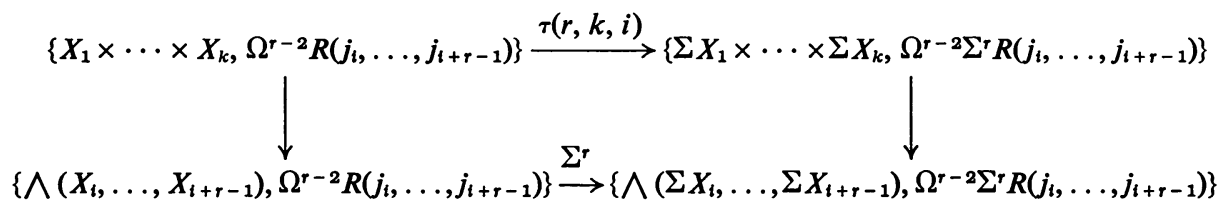

where the unlabeled arrows are projections.

Thus to prove the last assertion of the theorem it suffices to show that for integers $r$ and $k, 2 \leqq r \leqq k$, there is a map $\sigma(r, k)$ such that the following diagram commutes for each $i, 1 \leqq i \leqq k-r-1$.

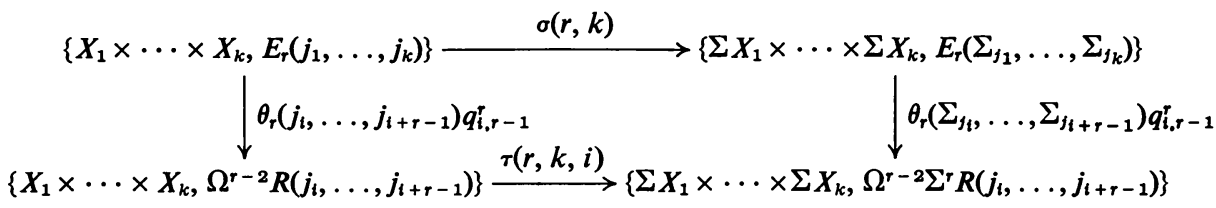

If $k=2$ or $r=2$ the existence of $\sigma(r, k)$ and the commutativity of the above diagram follow from the definition of $\Sigma \mathscr{R}$.

We assume inductively that $\sigma(r, k)$ is defined so that the above diagram commutes if $k<n$ and if $k=n$ and $r<m$.

Given $\psi: X_{1} \times \cdots \times X_{n} \rightarrow E_{m}\left(j_{1}, \ldots, j_{n}\right)$ let

$$
\begin{aligned}
& \psi_{0}: X_{1} \times \cdots \times X_{n} \rightarrow E_{m-1}\left(j_{1}, \ldots, j_{n}\right), \\
& \psi_{i}: X_{1} \times \cdots \times X_{n} \rightarrow P \Omega^{m-3} R\left(j_{i}, \ldots, j_{i+m-1}\right)
\end{aligned}
$$

for $1 \leqq i \leqq n-m+2$ be the compositions of $\psi$ and the canonical projections. Let

$$
\begin{aligned}
\tilde{\tau}(m-1, n, i):\left\{X_{1} \times \cdots \times X_{n}, P \Omega^{m-3} R\left(j_{i}, \ldots, j_{i+m-1}\right)\right\} \\
\rightarrow\left\{\Sigma X_{1} \times \cdots \times \Sigma X_{n}, P \Omega^{m-3} \Sigma^{m-1} R\left(j_{i}, \ldots, j_{i+m-1}\right)\right\}
\end{aligned}
$$

be defined similarly to $\tau$.

For any spaces $U$ and $V$ let $e:\{U, P V\} \rightarrow\{U, V\}$ be evaluation at the "endpoint". Then

$$
\tau(m-1, n, i)\left(e\left(\psi_{i}\right)\right)=e\left(\bar{\tau}(m-1, n, i)\left(\psi_{i}\right)\right) .
$$

Define $\sigma(m, n)(\psi)$ by $(\sigma(m, n)(\psi))_{0}=\sigma(m-1, n)(\psi)$ and

$$
\begin{aligned}
(\sigma(m, n)(\psi))_{i} & =\tau(m-1, n, i)\left(\psi_{i}\right), \quad 1 \leqq i \leqq n-m+2 . \\
e\left((\sigma(m, n)(\psi))_{i}\right) & =e\left(\tau(m-1, n, i)\left(\psi_{i}\right)\right) \\
& =\tau(m-1, n, i)\left(e\left(\psi_{i}\right)\right) \\
& =\tau(m-1, n, i)\left(\theta_{m-1}\left(j_{i}, \ldots, j_{i+m-2}\right) q_{i, m}^{m-1}{ }^{2} \psi\right) \\
& =\theta_{m-1}\left(\Sigma_{j_{i}}, \ldots, \Sigma_{j_{i+m+2}}\right) q_{i, m-2}^{m-1} \sigma(m-1, n)(\psi) \\
& =\theta_{m-1}\left(\Sigma_{j_{i}}, \ldots, \Sigma_{j_{i+m-2}}\right) q_{i, m-2}^{m-1}(\sigma(m, n) \psi)_{0}
\end{aligned}
$$

and thus $\sigma(m, n)(\psi): \Sigma X_{1} \times \cdots \times \Sigma X_{n} \rightarrow E_{m}\left(\Sigma_{j_{1}}, \ldots, \Sigma_{j_{n}}\right)$. It remains to show that 
the diagram commutes. To see this it suffices to consider the case $m=n$. Thus we must show that the following diagram commutes.

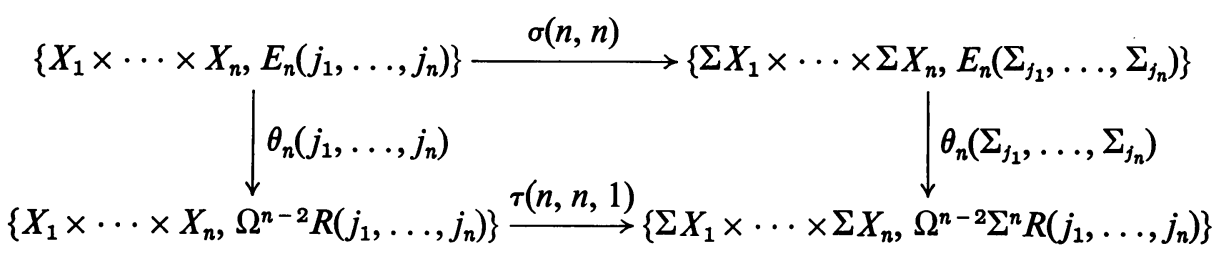

We check this for the " $p$ th face" of $\theta_{n}$. Let $\pi_{i}: E_{i} \rightarrow E_{i-1}$ be the projection. Let $h\left(\Sigma_{j_{1}}, \ldots, \Sigma_{j_{p}}\right)$ be as in $\S 2$.

Similarly

$$
\begin{aligned}
h\left(\Sigma_{j_{1}}, \ldots, \Sigma_{j_{p}}\right)(\sigma(n, n)(\psi)) & =\left(\sigma(p+1, n)\left(\pi_{p+2} \pi_{p+3} \cdots \pi_{n} \psi\right)\right)_{1} \\
& =\tau(p, n, 1)\left(\left(\pi_{p+2} \cdots \pi_{n} \psi\right)_{1}\right) \\
& =\tau(p, n, 1)\left(h\left(j_{1}, \ldots, j_{p}\right) \psi\right) .
\end{aligned}
$$

$$
h\left(\Sigma_{j_{p+1}}, \ldots, \Sigma_{j_{n}}\right)(\sigma(n, n)(\psi))=\bar{\tau}(n-p, n, p+1) h\left(j_{p+1}, \ldots, j_{n}\right) \psi .
$$

A direct calculation using the definition of $\Sigma \mathscr{R}$ shows

$$
\begin{aligned}
\left(\bar{\tau}(p, n, i)\left(h\left(j_{1}, \ldots, j_{p}\right) \psi\right)\right) \cdot(\bar{\tau}(n-p, n, p+1) & \left.\left(h\left(j_{p+1}, \ldots, j_{n}\right) \psi\right)\right) \\
& =\bar{\tau}(n, n, 1)\left(h\left(j_{1}, \ldots, j_{p}\right) \cdot h\left(j_{p+1}, \ldots, j_{n}\right) \psi\right) .
\end{aligned}
$$

Since this holds for each $p$ we have

$$
\theta_{n}\left(\Sigma_{j_{1}}, \ldots, \Sigma_{j_{n}}\right)(\sigma(n, n)(\psi))=\tau(n, n, 1)\left(\theta_{n}\left(j_{1}, \ldots, j_{n}\right) \psi\right)
$$

This completes the proof of the theorem.

CoROllaRY 3.15. In $\Sigma \mathscr{R}$ if $\left\{f_{1}, \ldots, f_{n}\right\}$ is defined then

$$
\Sigma_{*}^{n}\left\{f_{1}, \ldots, f_{n}\right\} \subset\left\{\Sigma f_{1}, \ldots, \Sigma f_{n}\right\} .
$$

Finally we study products involving higher products.

THEOREM 3.16. (a) Let $\varphi: X \rightarrow E_{n}\left(j_{1}, \ldots, j_{n}\right)$ be a map of type $\left(f_{1}, \ldots, f_{n}\right)$ and let $g: X \rightarrow R_{i}$. There exist maps

$$
\varphi_{g}: X \rightarrow E_{n}\left(j_{1}, \ldots,\left(j_{n}, i\right)\right) \text { and }{ }_{g} \varphi: X \rightarrow E_{n}\left(\left(i, j_{1}\right), j_{2}, \ldots, j_{n}\right)
$$

of type $\left(f_{1}, \ldots, f_{n} \cdot g\right)$ and $\left(g \cdot f_{1}, \ldots, f_{n}\right)$ respectively. Moreover $M_{n}\left(\varphi_{g}\right)=M_{n}(\varphi) \cdot g$ and $M_{n}\left({ }_{g} \varphi\right)=g \cdot M_{n}(\varphi)$.

(b) Let $\varphi: X_{1} \times \cdots \times X_{n} \rightarrow E_{n}\left(j_{1}, \ldots, j_{n}\right)$ be a map of type $\left(f_{1}, \ldots, f_{n}\right)$ and let $g: Y \rightarrow R_{i}$. There exist maps $\varphi_{g}: X_{1} \times \cdots \times \bigwedge\left(X_{n}, Y\right) \rightarrow E_{n}\left(j_{1}, \ldots,\left(j_{n}, i\right)\right)$ and ${ }_{g} \varphi: \bigwedge\left(Y, X_{1}\right) \times \cdots \times X_{n} \rightarrow E_{n}\left(\left(i, j_{1}\right), \ldots, j_{n}\right)$ of type $\left(f_{1}, \ldots, f_{n} \cdot g\right)$ and $\left(g \cdot f_{1}, \ldots, f_{n}\right)$ respectively. Moreover $T_{n}\left(\varphi_{g}\right)=T_{n}(\varphi) \cdot g$ and $T_{n}\left({ }_{g} \varphi\right)=g \cdot T_{n}(\varphi)$. (We consider the product to be from the smash product in $(\mathrm{b})$.)

Proof. The proof consists of multiplying the given liftings on the left or on the 
right by $g$ when they involve the first or last coordinate. The proof proceeds inductively and is straightforward. We omit the details.

Corollary 3.17. Let $f_{j}: X \rightarrow R_{j} j=1, \ldots, n$ and $g: X \rightarrow R_{i}$ then

(a) $\left\langle f_{1}, \ldots, f_{n}\right\rangle \cdot g \subset\left\langle f_{1}, \ldots, f_{n} \cdot g\right\rangle$,

(b) $g \cdot\left\langle f_{1}, \ldots, f_{n}\right\rangle \subset\left\langle g \cdot f_{1}, \ldots, f_{n}\right\rangle$.

Let $f_{j}: X_{j} \rightarrow R_{j}, j=1, \ldots, n$ and $g: Y \rightarrow R_{i}$ then

(c) $\left\{f_{1}, \ldots, f_{n}\right\} \cdot g \subset\left\{f_{1}, \ldots, f_{n} \cdot g\right\}$,

(d) $g \cdot\left\{f_{1}, \ldots, f_{n}\right\} \subset\left\{g \cdot f_{1}, \ldots, f_{n}\right\}$.

4. Chains for cohomology theories. Often in mathematics the motivation behind a definition is obscured by the time the definition and the ensuing theory appear in print. In this section we present the background which led us to formulate the definitions given above. In so doing we give the relationship between our work and that of Kraines.

In [3] Kraines defined higher Massey products in ordinary (singular) cohomology Our original goal was to extend these definitions to other cohomology theories. On "nice" categories cohomology theories are representable, i.e. given a cohomology theory $\mathscr{H}^{n}(X)$ there exists a spectrum, $\left\{Y_{n}\right\}$, such that $\mathscr{H}^{n}(X)=\left[X, Y_{n}\right]$ for all $n$.

Thus cohomology classes correspond to homotopy classes of maps $X \rightarrow Y_{n}$ and the set of maps $\left\{X, Y_{n}\right\}$ can be thought of as the "cocycles" of $\mathscr{H}^{n}(X)$. Now, two cocycles are cohomologous if their difference is a coboundary. Similarly two maps are homotopic if their difference is null-homotopic. Following this idea we define the $n$-cochains to be the set of null-homotopies, $\left\{X, P Y_{n+1}\right\}$. The coboundary is evaluation at the endpoint. Thus the set of cocycles is $\left\{X, \Omega Y_{n+1}\right\}$ which we assume equal to $\left\{X, Y_{n}\right\}$. Under the correct definition of "cycles mod boundaries" we then have $\mathscr{H}^{n}(X)=\left[X, Y_{n}\right]$.

Furthermore if $\left\{Y_{n}\right\}$ is a ringed spectrum, this operation can be used to define the cup product of cochains. In this setting our definition of the higher Massey product formally coincides with the definition given by Kraines.

For the sake of convenience we assume that the spectrum, $\mathscr{Y}=\left\{Y_{n}\right\}$ has the property that $\Omega Y_{n+1}=Y_{n}$ for all $n$.

Definition 4.1. $C^{n}(X, \mathscr{Y})=\left\{X, P Y_{n+1}\right\} . \delta: C^{n}(X, \mathscr{Y}) \rightarrow C^{n+1}(X, \mathscr{Y})$ is induced by the composite: $P Y_{n+1} \stackrel{e}{\rightarrow} Y_{n+1}=\Omega Y_{n+2} \subset P Y_{n+2}$ where $e$ is evaluation at the endpoint.

Clearly $\delta^{2}=*$ (the constant map). Set $\mathscr{Z}^{n}(X, \mathscr{Y})=\operatorname{ker} \delta$ and $\mathscr{B}^{n}(X, \mathscr{Y})=\operatorname{Im} \delta$. $\mathscr{Z}^{n}(X, \mathscr{Y})$ is therefore equal to $\left\{X, \Omega Y_{n+1}\right\}=\left\{X, Y_{n}\right\}$. Using the $H$-space structure of $\Omega Y_{n+1}, \mathscr{Z}^{n}(X, \mathscr{Y})$ is a monoid with an operation corresponding to the additive inverse.

Definition 4.2. $z, z^{\prime} \in \mathscr{Z}^{n}(X, \mathscr{Y})$ are said to be equivalent, $z \sim z^{\prime}$, if there exists a sequence of cocycles $z=z_{0}, z_{1}, \ldots, z_{n}=z^{\prime}$ such that $z_{i}-z_{i-1} \in \mathscr{B}^{n}(X, \mathscr{Y})$ for $i=1, \ldots, n$. 
Definition 4.3. $H^{n}(X, \mathscr{Y})=\mathscr{Z}^{n}(X, \mathscr{Y}) / \sim$.

One easily sees

Proposition 4.4. $H^{n}(X, \mathscr{Y})=\left[X, Y_{n}\right]$.

When constructing homotopy operations it is often useful to consider $C^{n}(X ; \mathscr{Y})$ $=\left\{X, P \Omega^{k} Y_{n+k+1}\right\}$ for $k>0$. To define the coboundary choose a relative homeomorphism, $\bar{w}:\left(I^{n-1}, I^{n-1}\right) \rightarrow\left(I^{n}, L^{n}\right)$. This induces $w:\left\{X, \Omega^{n-1} Y\right\} \rightarrow\left\{X, P \Omega^{n-2} Y\right\}$ given by

$$
(w f)\left(t_{1}, \ldots, t_{n-1}\right)=f(x) \bar{w}\left(t_{1}, \ldots, t_{n-1}\right) .
$$

$\delta^{\prime}$ is then the composite

$$
\left\{X, P \Omega^{n-1} Y_{k}\right\} \stackrel{e}{\longrightarrow}\left\{X, \Omega^{n-1} Y_{k}\right\} \stackrel{w}{\longrightarrow}\left\{X, P \Omega^{n-2} Y_{k}\right\}
$$

where $e$ is induced by restriction.

For $f \in\left\{X, P \Omega^{n-1} Y_{k}\right\},\left(f^{1}, \ldots, f^{n}\right)$ is a compatible set in $\left\{X, P \Omega^{n-2} Y_{k}\right\}$ and $\delta^{\prime} f=w\left(\sum_{1}^{n}(-1)^{i+1} f^{i}\right)$.

Now assume $\mathscr{Y}=\left\{Y_{k}\right\}$ is an associative ringed spectrum. As in $\S 1$ this pairing can be extended to give a product on the "cochain" level. Since $(f \cdot g)^{i}=f^{i} \cdot g$ for $i \leqq n$ and $(f \cdot g)^{j}=f \cdot g^{j-n}$ for $j>n$ we have on a formal basis

$$
\begin{aligned}
\delta^{\prime}(f \cdot g) & =w\left(\sum_{1}^{m+n}(-1)^{i+1}(f \cdot g)^{i}\right) \\
& =w\left(\left(\sum_{1}^{n}(-1)^{i+1} f^{i}\right) \cdot g\right)+w\left(\sum_{n+1}^{m+n}(-1)^{i+1} f \cdot(g)^{i-n}\right) \\
& =\left(\delta^{\prime} f\right) \cdot g+(-1)^{n} f \cdot\left(\delta^{\prime} g\right) .
\end{aligned}
$$

A lifting $\varphi$ of $X \rightarrow R_{j_{1}} \times \cdots \times R_{j_{n}}$ of type $\left(u_{1}, \ldots, u_{n}\right)$ to $E_{n}\left(j_{1}, \ldots, j_{n}\right)$ is a choice of null-homotopies in $X$, i.e. cochains. Let $h\left(j_{i}, \ldots, j_{k}\right)$ be as in $\S 2$. The set of nullhomotopies

$$
\left\{h\left(j_{i}, \ldots, j_{k}\right) \varphi \mid 1 \leqq i \leqq k \leqq n \text { and }(i, k) \neq(1, n)\right\}
$$

corresponds formally to what Kraines calls "a defining system for the (cochain) product $\left\langle u_{1}, \ldots, u_{n}\right\rangle$ " (Definition 1, p. 431 of [3]). Under this correspondence our definition

$$
M_{n}(\varphi)=\Sigma(-1)^{k+1}\left(h\left(j_{1}, \ldots, j_{k}\right) \varphi\right) \cdot\left(h\left(j_{k+1}, \ldots, j_{n}\right) \varphi\right)
$$

is formally the same as the definition given by Kraines.

5. Construction of the higher products-Homotopy associative case. The construction of the universal higher product in $\$ 2$ depended upon the fact that the pairing of the ringed set was strictly associative. If the pairing is only homotopy associative a different construction is necessary and, in fact, one may be unable to to define higher products. Whereas in the associative case the construction consisted of 'gluing together' $(n-1)$ maps, the construction given in this section 
consists of 'gluing together' $2^{n-1}-1$ maps. We present the outline of the general procedure.

Stasheff, in his study of homotopy associativity of $H$-spaces [8], introduced the notion of $A_{n}$ forms and $A_{n}$ spaces. These forms measure the degree of homotopy associativity of an $H$ space, $G$, and are the obstructions to constructing a classifying space for $G$. The notion of $A_{n}$ forms carry over to ringed sets mutatis mutandis. We recall some details.

The spaces $K_{n}, n \geqq 2$ on which the $A_{n}$ forms are defined are constructed as follows. Consider a word with $i$ letters, $x_{1} \cdots x_{i}$. Corresponding to each nontrivial insertion of parentheses (in this word) there is a cell on the boundary of $K_{i}$. If the parentheses enclose $x_{k} \cdots x_{k+s-1}$ we regard the cell as a homeomorph of $K_{r} \times K_{s}$ $(r+s=i+1)$ under a map which is called $\partial_{k}(r, s)$. Two such cells intersect only on their boundary and the cells of the intersection correspond to the insertion of a second pair of parentheses. Start with $K_{2}=*$. Given $K_{2}, \ldots, K_{i-1}$ construct the boundary of $K_{i}$ by fitting together copies of $K_{r} \times K_{s}$ as indicated above. Set $K_{i}$ equal to the cone on the boundary.

Proposition 5.1 (Stasheff). $K_{i} \approx I^{i-2}$.

Definition 5.2. A ringed set $\mathscr{R}$ admits an $A_{n}$ structure if there are maps

$$
M_{i}: K_{i} \times R_{j_{1}} \times \cdots \times R_{j_{i}} \rightarrow R\left(j_{1}, \ldots, j_{i}\right)
$$

for $2 \leqq i \leqq n$ and all distinguished $i$-tuples $\left(j_{1}, \ldots, j_{i}\right)$ such that

(1) $M_{2}=\mu: * \times R_{i} \times R_{j} \rightarrow R(i, j)$.

(2) For $\rho \in K_{r}, \sigma \in K_{s}(r+s=i+1)$

$$
\begin{aligned}
M_{i}\left(\partial_{k}(r, s)(\rho, \sigma), x_{1}, \ldots,\right. & \left.x_{i}\right) \\
& =M_{r}\left(\rho, x_{1}, \ldots, x_{k-1}, M_{s}\left(\sigma, x_{k}, \ldots, x_{k+s-1}\right), x_{k+s}, \ldots, x_{i}\right) .
\end{aligned}
$$

Such a system of maps is called an $A_{n}$ form on $\mathscr{R}$. A ringed set together with an $A_{n}$ form is called an $A_{n}$ ringed set.

An $\dot{A}_{3}$ ringed set is a ringed set $\mathscr{R}=\left\{R_{j}, \mu\right\}$ together with a homotopy

$$
M_{3}: \mu(\mu \times 1) \sim \mu(1 \times \mu) .
$$

If $\mathscr{R}$ is an $A_{n}$ ringed set, $n$-fold products may be defined.

The universal examples $E_{r}\left(j_{1}, \ldots, j_{n}\right)$ are defined as in $\S 2$. The change here is in the definition of $\theta_{n}\left(j_{1}, \ldots, j_{n}\right)$. We first construct model spaces $W_{j}$ such that the set of maps $f: \dot{W}_{j} \rightarrow R$ under the compact open topology is our model for $\Omega^{j-2} R$.

Let $x_{1} \cdots x_{n}$ be a word with $n$ letters. Corresponding to each nontrivial partition of $x_{1} \cdots x_{n}$ there is a cell on the boundary of $W_{n}$. In particular if $x_{1} \cdots x_{n}$ is partitioned into $t$ parts with $r_{i}$ letters in the $i$ th part, the corresponding cell is a homeomorph of $K_{t} \times W_{r_{1}} \times \cdots \times W_{r_{t}}$ under a map $\partial\left(r_{1}, \ldots, r_{t}\right)$.

The cells on the boundary of $K_{t} \times W_{r_{1}} \times \cdots \times W_{r_{t}}$ correspond to two operations on the partition of $x_{1} \cdots x_{n}$. 
(a) a nontrivial insertion of parentheses subject to the condition that the parentheses may not further subdivide any part, and

(b) further subdivision of a given part with the subdivided part contained in parentheses.

EXAMPLE. $x_{1}\left|x_{2} x_{3} x_{4}\right| x_{5} \mid x_{6} x_{7}$.

Cells of type (a).

Cells of type (b).

$$
\begin{aligned}
& \left(x_{1} \mid x_{2} x_{3} x_{4}\right)\left|x_{5}\right| x_{6} x_{7} \\
& x_{1}\left|\left(x_{2} x_{3} x_{4} \mid x_{5}\right)\right| x_{6} x_{7} \\
& x_{1}\left|x_{2} x_{3} x_{4}\right|\left(x_{5} \mid x_{6} x_{7}\right) \\
& \left(x_{1}\left|x_{2} x_{3} x_{4}\right| x_{5}\right) \mid x_{6} x_{7} \\
& x_{1} \mid\left(x_{2} x_{3} x_{4}\left|x_{5}\right| x_{6} x_{7}\right)
\end{aligned}
$$

$$
\begin{aligned}
& x_{1}\left|\left(x_{2} \mid x_{3} x_{4}\right)\right| x_{5} \mid x_{6} x_{7} \\
& x_{1}\left|\left(x_{2} x_{3} \mid x_{4}\right)\right| x_{5} \mid x_{6} x_{7} \\
& x_{1}\left|\left(x_{2}\left|x_{3}\right| x_{4}\right)\right| x_{5} \mid x_{6} x_{7} \\
& x_{1}\left|x_{2} x_{3} x_{4}\right| x_{5} \mid\left(x_{6} \mid x_{7}\right)
\end{aligned}
$$

The cell of type (a) correspond to the cells contained in $\dot{K}_{t} \times W_{r_{1}} \times \cdots \times W_{r_{t}}$ while the cells of type (b) are contained in $U K_{t} \times W_{r_{1}} \times \cdots \times \dot{W}_{r_{i}} \times \cdots \times W_{r_{t}}$. Two cells in the boundary of $W_{n}$ meet only on their boundaries. Here two cells are identified if their related words (with partitions and parentheses) agree.

Explicitly, we identify

$$
\partial\left(r_{1}, \ldots, r_{m+n}\right)\left(\partial_{i}(m, n)(\sigma, \rho), x_{1}, \ldots, x_{m+n}\right)
$$

with

$$
\begin{aligned}
\partial\left(r_{1}, \ldots, r_{i-1}, R, r_{i+n}, \ldots, r_{m+n}\right) & \left(\sigma, x_{1}, \ldots, x_{i-1},\right. \\
& \left.\partial\left(r_{i}, \ldots, r_{i+n+1}\right)\left(\rho, x_{i}, \ldots, x_{i+n-1}\right), x_{i+n}, \ldots, x_{m+n}\right)
\end{aligned}
$$

where $R=\sum_{j=i}^{i+n-1} r_{j}$.

Start with $W_{1}=*$. Given $W_{1}, \ldots, W_{n-1}$ construct the boundary of $W_{n}$ by fitting together homeomorphs of $K_{t} \times W_{r_{1}} \times \cdots \times W_{r_{t}}$ as indicated above. Set $W_{n}$ equal to the cone of the boundary.

We assert that $W_{n} \approx I^{n-1}$. We have checked this for $n \leqq 5$. To verify this for all $n$ one must construct a model for $W_{n}$. Since there are $2^{n-1}-1$ cells in the boundary, each of which is itself complicated this is a nontrivial combinatorial problem. We indicate below the construction for $n \leqq 4$. (See figure on following page.)

Define $\theta_{n}\left(j_{1}, \ldots, j_{n}\right): E_{n}\left(j_{1}, \ldots, j_{n}\right) \rightarrow\left(R\left(j_{1}, \ldots, j_{n}\right)\right)^{\dot{W}_{n}}$ by

$$
\begin{aligned}
\theta_{n}\left(j_{1}, \ldots, j_{n}\right)(x) \partial\left(r_{1}, \ldots,\right. & \left.r_{t}\right)\left(k, \sigma_{1}, \ldots, \sigma_{t}\right) \\
& =M_{t}\left(k, h\left(j_{1}, \ldots, j_{r_{1}}\right)(x)\left(\sigma_{1}\right), \ldots, h\left(j_{n-r_{t}+1}, \ldots, j_{n}\right)(x)\left(\sigma_{t}\right)\right)
\end{aligned}
$$

where $\left(k, \sigma_{1}, \ldots, \sigma_{t}\right) \in K_{t} \times W_{r_{1}} \times \cdots \times W_{r_{t}}$ and $h\left(j_{i}, \ldots, j_{s}\right)$ is as in $\S 2$ and we understand $h\left(j_{i}\right)(x)\left({ }^{*}\right)=h\left(j_{i}\right)(x)$. 
$x_{1} \mid x_{2}$

$W_{2}$
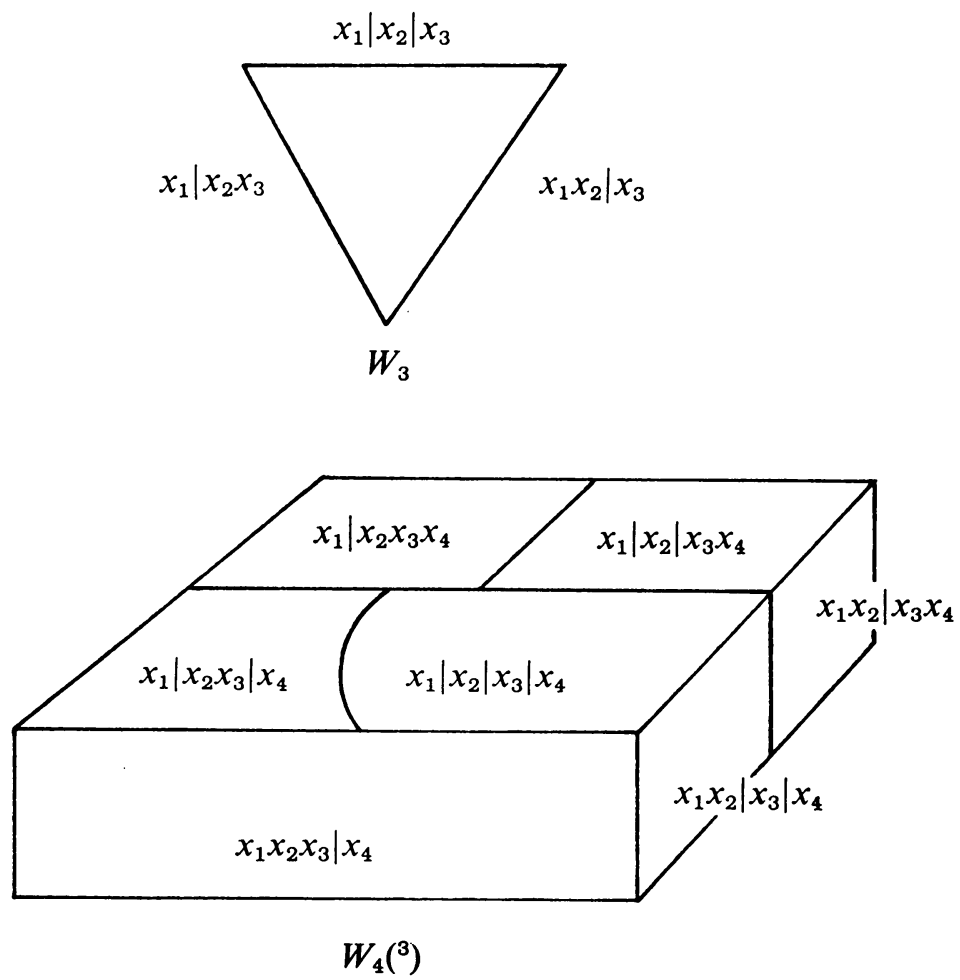

The verification that $\theta_{n}$ is well defined is straightforward and follows from the definition of the $A_{n}$ forms and the construction of the $W_{i}$ 's.

$M_{n}(\varphi), T_{n}(\varphi),\left\langle f_{1}, \ldots, f_{n}\right\rangle,\left\{f_{1}, \ldots, f_{n}\right\}$ are defined exactly as in $\S 2$.

Theorems 3.1, 3.6, 3.7, 3.9, and 3.10 carry over to this case with no difficulty. The definitions of morphism of ringed sets and linearity must be changed to appropriate definitions in the category of $A_{n}$ ringed sets. Under these new definitions Theorems 3.2, 3.4, and 3.14 hold. We leave the details to the reader.

6. Higher commutator products. Since the commutator product

$$
\langle 1,1\rangle: \Omega X \times \Omega X \rightarrow \Omega X
$$

is neither associative nor homotopy associative, the constructions of $\$ \$ 2$ and 3 are

(3) All hidden faces are identified to * the base point. 
not applicable. In this section we construct higher commutator products in loop spaces. The methods used here are a continuation of those used in the earlier parts of this paper. A different approach is taken by Williams [10].

Set $\Omega^{n} Y=\left\{f: I^{n+1} \rightarrow Y\right\}, \bar{\Omega} Y=\{f:(I, I) \rightarrow(Y, *)\}$ and $P \Omega^{n} Y=\left\{f: I^{n+1} \rightarrow Y\right\}$. We recall that the commutator, $\langle 1,1\rangle: \bar{\Omega} X \times \bar{\Omega} X \rightarrow \Omega X$ is defined by

$$
\begin{aligned}
\langle 1,1\rangle\left(l_{1}, l_{2}\right)\left(t_{1}, t_{2}\right) & =l_{1}\left(t_{1}\right) \quad \text { if } t_{2}=0,1, \\
& =l_{2}\left(t_{2}\right) \quad \text { if } t_{1}=0,1 .
\end{aligned}
$$

Throughout this section we shall use many copies of $\Omega^{j} X$. To distinguish between them we shall index them, e.g. $\Omega^{j} X\left(k_{1}, \ldots, k_{j}\right)$. It will be clear from the context which copy is referred to. $\theta_{2}(i, j): \bar{\Omega} X(i) \times \bar{\Omega} X(j) \rightarrow \Omega X(i, j)$ is the commutator, $\langle 1,1\rangle$, defined above.

Set $E_{n}^{2}(X)=\bar{\Omega} X(1) \times \cdots \times \bar{\Omega} X(n)$ and let $q(i, j): E_{n}^{2}(X) \rightarrow \bar{\Omega} X(i) \times \bar{\Omega} X(j)$ be the projection. We assume inductively that $E_{k}^{j}(X), j \leqq k$ is defined for $k<n$ and for $j<m$ wben $k=n$, and $\theta_{j}: E_{j}^{j}(X) \rightarrow \Omega^{j-1} X$ is defined for $j<n$ such that:

(a) For each $j$-tuple $\left(i_{1}, \ldots, i_{j}\right), 1 \leqq i_{1}<\cdots<i_{j} \leqq k$ there is a projection $q\left(i_{1}, \ldots, i_{j}\right): E_{k}^{j}(X) \rightarrow E_{j}^{j}(X)$ for all $j$ and $k$ for which $E_{k}^{j}(X)$ is defined.

(b) $E_{k}^{j}(X)$ is the fibre space induced from the canonical path fibration by

$$
\Pi \theta_{j-1} q\left(i_{1}, \ldots, i_{j-1}\right): E_{k}^{j-1} \rightarrow \Pi \Omega^{j-2} X\left(i_{1}, \ldots, i_{j-1}\right)
$$

where the product runs over all $(j-1)$-tuples, $\left(i_{1}, \ldots, i_{j-1}\right)$, such that

$$
1 \leqq i_{1}<\cdots<i_{j-1} \leqq k \text {. }
$$

(c) For $j<n, \theta_{j}(x)\left(t_{1}, \ldots, t_{j}\right)=h_{j}(1, \ldots, \hat{l}, \ldots, j)$ if $t_{i}=0,1$ where, for $j>1$, $h_{k}\left(i_{1}, \ldots, i_{j}\right): E_{k}^{k}(X) \rightarrow P \Omega^{j-2} X\left(i_{1}, \ldots, i_{j}\right)$ is the composite of projections,

$$
E_{k}^{k}(X) \rightarrow E_{k}^{k-1} \rightarrow \cdots \rightarrow E_{k}^{j+1}(X) \rightarrow P \Omega^{j-2} X\left(i_{1}, \ldots, i_{j}\right)
$$

and $h\left(i_{j}\right)$ is the composite, $E_{k}^{k}(X) \rightarrow \cdots \rightarrow E_{k}^{2}(X) \rightarrow \bar{\Omega} X\left(i_{j}\right)$.

Definition 6.1. (a) $E_{n}^{m}(X)$ is the fibre space induced from the canonical path fibration by

$$
\Pi \theta_{m-1} q\left(i_{1}, \ldots, i_{m-1}\right): E_{n}^{m-1}(X) \rightarrow \Pi \Omega^{m-2} X\left(i_{1}, \ldots, i_{m-1}\right)
$$

where the product runs over all $(m-1)$-tuples, $\left(i_{1}, \ldots, i_{m-1}\right)$, such that

$$
1 \leqq i_{1}<\cdots<i_{m-1} \leqq n .
$$

(b) The projections, $q\left(i_{1}, \ldots, i_{m}\right): E_{n}^{m}(X) \rightarrow E_{m}^{m}(X)$, are induced by the obvious projections from the cartesian product.

(c) The universal $n$-fold commutator, $\theta_{n}: E_{n}^{n}(X) \rightarrow \Omega^{n-1} X$, is defined by

$$
\theta_{n}(x)\left(t_{1}, \ldots, t_{n}\right)=h_{n}(1, \ldots, \hat{\imath}, \ldots, n)(x)\left(t_{1}, \ldots, \hat{t}_{i}, \ldots, t_{n}\right)
$$

for $t_{i}=0,1$.

One easily verifies that this defines a map $E_{n}^{n}(X) \rightarrow \Omega^{n-1} X$. Extend the above notation and let $E_{n}^{n+1}(X)$ denote the fibre space induced from $E_{n}^{n}(X)$ by $\theta_{n}$. 
Following the notation of [5] let $T_{i}\left(X_{1}, \ldots, X_{n}\right)$ be the subspace of $X_{1} \times \cdots \times X_{n}$ consisting of those $n$-tuples with at least $i$ coordinates at base points. $T_{0}$ is the cartesian product, $T_{1}$ is the "fat" wedge and $T_{n-1}\left(X_{1}, \ldots, X_{n}\right)$ is the one point union. If $X_{1}=X_{2}=\cdots=X_{n}=X$ we denote $T_{i}\left(X_{1}, \ldots, X_{n}\right)$ by $T_{i}^{n}(X)$.

Let $\left(I^{n}\right)^{(i)}$ be the $i$ skeleton of $I^{n}$ and set $\rho: I^{n} \rightarrow T_{0}^{n}\left(S^{1}\right)$ equal to the quotient map obtained by identifying opposite faces. Clearly $\rho\left(\left(I^{n}\right)^{(i)}\right)=T_{n-i}^{n}\left(S^{1}\right)$.

THEOREM 6.2. (a) $E_{n}^{i}(X)=X^{T_{n-i+1}^{n}\left(S^{1}\right)}$,

(b) $\theta_{n}: E_{n}^{n}(X) \rightarrow \Omega^{n-1} X$ is the map induced by $\rho \mid I^{n}$ under the identifications of part (a).

Proof. (a) We show that there is a 1-1 correspondence between the points of $E_{n}^{i}(X)$ and maps $T_{n-i+1}^{n}\left(S^{1}\right) \rightarrow X$. We leave the study of the point set topology to the reader.

For all $n \geqq 2, E_{n}^{2}(X)=(\bar{\Omega} X)^{n}$ and $X^{T_{n-1}^{n}\left(S^{1}\right)}=X^{S^{1} \vee \ldots \vee S^{1}}$. Each of these is in 1-1 correspondence with the set of $n$-tuples $\left(f_{1}, \ldots, f_{n}\right)$, where $f_{i}: S^{1} \rightarrow X$. $\theta_{2} \in \Omega X$ is the composite,

$$
I^{2} \stackrel{\rho}{\longrightarrow} S^{1} \vee S^{1} \stackrel{f_{1} \mid f_{2}}{\longrightarrow} X .
$$

We assume inductively that the theorem is true for $E_{j}^{i}$ when either $j<n$ or when $j=n$ and $i<k$. Thus we must show $E_{n}^{k}(X)=X^{T_{n-k+1}^{n}\left(S^{1}\right)}$.

$E_{n}^{k}(X)$ is the subset of $E_{n}^{k-1} \times \Pi P \Omega^{k-2} X\left(i_{1}, \ldots, i_{k-1}\right)$ consisting of $\left(\left(k_{k-1}^{n}\right)+1\right)-$ tuples $\left(\varphi, \Pi_{\eta}\left(i_{1}, \ldots, i_{k-1}\right)\right)$ where the product is taken over all $1 \leqq i_{1}<\cdots<$ $i_{k-1} \leqq n$ and

$$
\eta\left(i_{1}, \ldots, i_{k-1}\right) \mid I^{k-1}=\theta_{k-1} q\left(i_{1}, \ldots, i_{k-1}\right)(\varphi) .
$$

By induction $\varphi$ corresponds to a map $\tilde{\varphi}: T_{n-k+2}^{n}\left(S^{1}\right) \rightarrow X$ and hence

$$
\tilde{\varphi} \rho:\left(I^{n}\right)^{(k-2)} \rightarrow X .
$$

For $1 \leqq i_{1}<\cdots<i_{k-1} \leqq n, \rho^{-1}\left(S_{i_{1}}^{1} \times \cdots \times S_{i_{k-1}}^{1}\right)$ consists of the $2^{n-k+1}(k-1)$ faces of $I^{n}$ on which the coordinates in the $\left(i_{1}, \ldots, i_{k-1}\right)$ plane vary and the remainder are constant $(0$ or 1$)$. We let $I_{i_{1}} \times \cdots \times I_{i_{k-1}}$ represent these faces. An extension of $\varphi$ to $S_{i_{1}}^{1} \times \cdots \times S_{i_{k-1}}^{1} \subset T_{n-k+1}^{n}\left(S^{1}\right)$ is given by an extension of $\tilde{\varphi} \rho$ to $I_{i_{1}} \times \cdots \times I_{i_{k-1}}$. Such a map extends

$$
\tilde{\varphi} \rho \mid \partial\left(I_{i_{1}} \times \cdots \times I_{i_{k-1}}\right)=\theta_{k} q\left(i_{1}, \ldots, i_{k-1}\right)(\varphi)
$$

and is given by $\eta\left(i_{1}, \ldots, i_{k-1}\right) \in P \Omega^{k-2} X\left(i_{1}, \ldots, i_{k-1}\right)$. Conversely, an extension of $\varphi$ to $S_{i_{1}}^{1} \times \cdots \times S_{i_{k-1}}^{1}$ determines

$$
\eta\left(i_{1}, \ldots, i_{k-1}\right) \in P \Omega^{k-2} X\left(i_{1}, \ldots, i_{k-1}\right)
$$

which restricted to $I^{k-1}$ is $\theta_{k} q\left(i_{1}, \ldots, i_{k-1}\right)(\varphi)$. This is true for each $(k-1)$-tuple $\left(i_{1}, \ldots, i_{k-1}\right)$. Hence maps $T_{n-k+1}^{n}\left(S^{1}\right) \rightarrow X$ are in 1-1 correspondence with the points of $E_{n}^{k}(X)$. 
Furthermore under this correspondence $\theta_{n}(\varphi)$ is the composite

$$
I^{n-1} \stackrel{\rho}{\longrightarrow} T_{1}^{n}\left(S^{1}\right) \stackrel{\tilde{\varphi}}{\longrightarrow} X
$$

and part (b) follows. A proof similar to the above establishes the theorem when $k=n+1$.

COROLlaRY 6.3. $\theta_{n}$ is null homotopic if and only if the fibration,

$$
i^{\#}: X^{T_{0}^{n}\left(S^{1}\right)} \rightarrow X^{T_{1}^{n}\left(S^{1}\right)}
$$

has a cross-section. $\left(i: T_{1}^{n}\left(S^{1}\right) \rightarrow T_{0}^{n}\left(S^{1}\right)\right.$ is inclusion. $)$

THEOREM 6.4. If $X$ is an H-space, $\theta_{n}$ is null-homotopic.

Proof. We construct a map

$$
s: X^{T_{1}^{n}\left(S^{1}\right)} \rightarrow X^{T_{0}^{n}\left(S^{1}\right)}
$$

such that $i^{\#} S \sim 1 . s$ is defined to be the following composite

$$
X^{T_{1}^{n}\left(S^{1}\right)} \rightarrow(\Omega \Sigma X)^{\Omega \Sigma T_{1}^{n}\left(S^{1}\right)} \rightarrow(\Omega \Sigma X)^{\Omega \Sigma T_{0}^{n}\left(S^{1}\right)} \rightarrow(\Omega \Sigma X)^{T_{0}^{n}\left(S^{1}\right)} \rightarrow X^{T_{0}^{n}\left(S^{1}\right)}
$$

where the first map is induced by the functor $\Omega \Sigma$, the second is induced by $\Omega r$ where $r$ is a retraction $\Sigma T_{0}^{n}\left(S^{1}\right) \rightarrow \Sigma T_{1}^{n}\left(S^{1}\right)$, the next map is induced by the adjoint of the identity $T_{0}^{n}\left(S^{1}\right) \rightarrow \Omega \Sigma T_{0}^{n}\left(S^{1}\right)$, and the last map is induced by a map $\Omega \Sigma X \rightarrow X$ such that $X \rightarrow \Omega \Sigma X \rightarrow X$ is homotopic to the identity. Such a map exists since $X$ is an $H$-space. The proof that $i^{\#} \sim 1$ is routine (see the proof of Theorem 2.5 in [5]).

Using the universal commutator we now define the analogues of the Massey and Toda products.

Definition 6.5. Given $\varphi: Y \rightarrow E_{n}^{n}(X)$ define the $n$-fold commutator, $C_{n}(\varphi)$ $=\varphi^{*}\left[\theta_{n}\right] \in\left[Y, \Omega^{n-1} X\right]$. We say $\varphi$ is of type $\left(f_{1}, \ldots, f_{n}\right)$ if $\varphi$ covers

$$
\left(f_{1} \times \cdots \times f_{n}\right) \Delta^{n}: Y \rightarrow(\bar{\Omega} X)^{n} .
$$

( $\Delta^{n}$ is the diagonal.) The set of commutators of type $\left(f_{1}, \ldots, f_{n}\right)$ is defined by

$$
C\left(f_{1}, \ldots, f_{n}\right)=\left\{C_{n}(\varphi) \mid \varphi \text { is of type }\left(f_{1}, \ldots, f_{n}\right)\right\} .
$$

These are the commutators arising from the group structure of $[Y, \Omega X]$.

Proposition 6.6. If $X$ is an $H$ space or $Y$ is a suspension then $C_{n}(\varphi)=0$.

Proof. If $Y=\Sigma Y^{\prime}$ and $\varphi: Y \rightarrow E_{n}^{n}(X)$, there is an adjoint $\bar{\varphi}: Y^{\prime} \rightarrow E_{n}^{n}(\Omega X)$ such that the adjoint of $C_{n}(\varphi)$ is

$$
C_{n}(\bar{\varphi}) \in\left[Y^{\prime}, \Omega(\Omega X)\right] .
$$

This reduces the problem to that of the range space being the loop space of an $H$-space. The proposition is then a corollary to Theorem 6.4. 
To define the higher Samelson products we again need the notion of a special map.

Definition 6.7. $\varphi: Y_{1} \times \cdots \times Y_{n} \rightarrow E_{n}^{n}(X)$ is special if for each $k$-tuple $\left(i_{1}, \ldots, i_{k}\right), 1 \leqq i_{1}<\cdots<i_{k} \leqq n$ and $k<n$, there is a map

$$
\varphi\left(i_{1}, \ldots, i_{k}\right): Y_{i_{1}} \times \cdots \times Y_{i_{k}} \rightarrow E_{n}^{k+1}(X)
$$

such that the following diagram commutes.

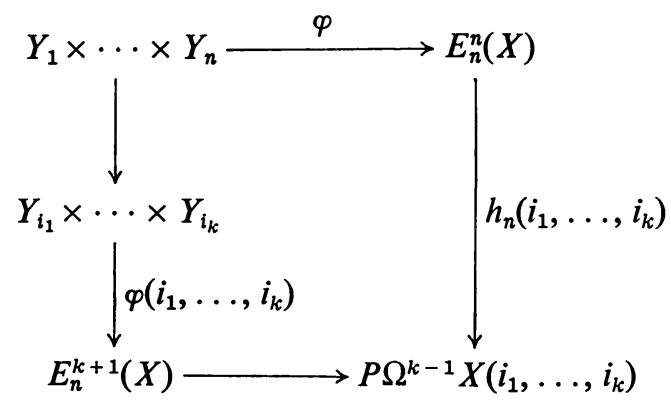

where the unlabeled maps are canonical projections.

Definition 6.8. Given a special map $\varphi: Y_{1} \times \cdots \times Y_{n} \rightarrow E_{n}^{n}(X)$ define $S_{n}(\varphi)$ the $n$-fold Samelson product by

$$
S_{n}(\varphi)=q\left(\varphi^{*}\left[\theta_{n}\right]\right) \in\left[\bigwedge\left(Y_{1}, \ldots, Y_{n}\right), \Omega^{n-1} X\right]
$$

where $q$ is the projection onto a direct summand as in Definition 2.5. We say $\varphi$ is of type $\left(f_{1}, \ldots, f_{n}\right)$ if $\varphi$ covers

$$
\left(f_{1} \times \cdots \times f_{n}\right): Y_{1} \times \cdots \times Y_{n} \rightarrow(\bar{\Omega} X)^{n} .
$$

The set of $n$-fold Samelson products of type $\left(f_{1}, \ldots, f_{n}\right)$ is defined by

$$
\left\langle f_{1}, \ldots, f_{n}\right\rangle=\left\{S_{n}(\varphi) \mid \varphi \text { is special of type }\left(f_{1}, \ldots, f_{n}\right)\right\} .
$$

Proposition 6.9. If $X$ is an $H$-space, $S_{n}(\varphi)=0$.

Proof. Theorem 6.4.

The following naturality properties are easily proven.

THEOREM 6.10. (a) If $g: W \rightarrow Y, C_{n}(\varphi g)=g^{*} C_{n}(\varphi)$.

(b) $g^{*} C_{n}\left(f_{1}, \ldots, f_{n}\right) \subset C_{n}\left(f_{1} g, \ldots, f_{n} g\right)$.

(c) If $g_{i}: W_{i} \rightarrow Y_{i}, i=1, \ldots, n$,

$$
\left(\bigwedge\left(g_{1}, \ldots, g_{n}\right)\right)^{*} S_{n}(\varphi)=S_{n}\left(\varphi\left(g_{1} \times \cdots \times g_{n}\right)\right) .
$$

(d) $\left(\bigwedge\left(g_{1}, \ldots, g_{n}\right)\right)^{*}\left\langle f_{1}, \ldots, f_{n}\right\rangle \subset\left\langle f g_{1}, \ldots, f g_{n}\right\rangle$. 
TheOREM 6.11. Let $\psi: X \rightarrow U$. $\psi$ induces $E_{n}^{n}(\psi): E_{n}^{n}(X) \rightarrow E_{n}^{n}(U)$ such that the following diagram commutes for all $n$.

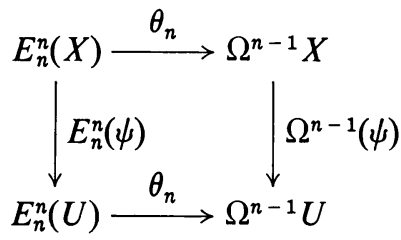

Given $f: \Omega X \rightarrow \Omega U$ it does not necessarily induce a map $E_{n}^{n}(X) \rightarrow E_{n}^{n}(U)$ with the above properties. If $f(x y) \sim f(x) f(y), f$ induces a map $E_{2}^{2}(X) \rightarrow E_{2}^{2}(U)$ such that the above diagram homotopy commutes but may not induce a map $E_{3}^{3}(X) \rightarrow E_{3}^{3}(U)$. We define the commutative degree of $f$ by setting $\operatorname{cd}(f)>n$ if there is an induced map $E_{n}^{n}(X) \rightarrow E_{n}^{n}(U)$ such that the diagram given above homotopy commutes. Theorem 6.11 can then be restated as $\operatorname{cd}(\Omega f)=\infty$.

Consider $Q\left(Y_{1}, \ldots, Y_{n}\right)=\bigcup_{j=1}^{n} C Y_{1} \times \cdots \times Y_{j} \times \cdots \times C Y_{n}$ contained in $C Y_{1} \times \cdots \times C Y_{n}$ where $C Y$ is the cone on $Y . Q\left(Y_{1}, \ldots, Y_{n}\right)$ is a model for

$$
\Sigma^{n-1} \wedge\left(Y_{1}, \ldots, Y_{n}\right)
$$

Let

$$
w: Q\left(Y_{1}, \ldots, Y_{n}\right) \rightarrow T_{1}\left(\Sigma Y_{1}, \ldots, \Sigma Y_{n}\right)
$$

be the map which identifies $Y_{i} \subset C Y_{i}$ to a point for all $i$. Given

$$
\varphi: T_{1}\left(\Sigma Y_{1}, \ldots, \Sigma Y_{n}\right) \rightarrow X,
$$

$\varphi_{*}[w]=W(\varphi)$ is the $n$th order Whitehead product studied in [5].

The adjoint map, $\alpha_{n-1}:\left\{\Sigma^{n-1} A, B\right\} \rightarrow\left\{A, \Omega^{n-1} B\right\}$ is defined by $\left(\alpha_{n-1}(f)\right)(a)(\lambda)$ $=f(\lambda, a)$ for $\lambda \in I^{n}$.

THEOREM 6.12. There is a 1-1 correspondence between special maps, $\varphi: Y_{1} \times \cdots \times Y_{n} \rightarrow E_{n}^{n}(X)$ and maps $\psi: T_{1}\left(\Sigma Y_{1}, \ldots, \Sigma Y_{n}\right) \rightarrow X$ such that $\left(\alpha_{n-1}\right)_{*} W(\psi)=S_{n}(\varphi)$.

Proof. By Theorem 6.3 and adjointness there is a 1-1 correspondence between maps $\varphi: Y_{1} \times \cdots \times Y_{n} \rightarrow E_{n}^{n}(X)$ and maps $\tilde{\varphi}: Y_{1} \times \cdots \times Y_{n} \times T_{1}^{n}\left(S^{1}\right) \rightarrow X$. $\tilde{\varphi}$ determines a map $T_{1}\left(\Sigma Y_{1}, \ldots, \Sigma Y_{n}\right) \rightarrow X$ if and only if $\varphi$ is special. Thus the first part of the theorem is proven.

To prove the last assertion of the theorem let $\varphi: Y_{1} \times \cdots \times Y_{n} \rightarrow E_{n}^{n}(X)$ be special and let $\psi: T_{1}\left(\Sigma Y_{1}, \ldots, \Sigma Y_{n}\right) \rightarrow X$ be the map corresponding to $\varphi$.

$$
\begin{aligned}
\alpha_{n-1} W(\psi)\left(t_{1}, y_{1}, \ldots, t_{n}, y_{n}\right) & =\alpha_{n-1}(\psi w)\left(t_{1}, y_{1}, \ldots, t_{n}, y_{n}\right) \\
& =\varphi\left(y_{1}, \ldots, y_{n}\right)\left(t_{1}, \ldots, t_{n}\right) \\
& =\theta_{n}\left(\varphi\left(y_{1}, \ldots, y_{n}\right)\right)\left(t_{1}, \ldots, t_{n}\right) \\
& =S_{n}(\varphi)\left(y_{1}, \ldots, y_{n}\right)\left(t_{1}, \ldots, t_{n}\right) .
\end{aligned}
$$

We conclude this section by discussing several notions of higher commutativity of a loop space. We first recall that the Whitehead product order of $X$, WP $(X)$, 
was defined in [6] to be the largest integer $n$ such that all $(n-1)$-fold generalized Whitehead products were zero in the homotopy of $X$. We can also define wp $(X)$ to be the least integer $n$, such that there are maps $f_{i}: \Sigma Y_{i} \rightarrow X, i=1, \ldots, n$, which have the property that there is no map $\Sigma Y_{1} \times \cdots \times \Sigma Y_{n} \rightarrow X$ of type $\left(f_{1}, \ldots, f_{n}\right)$. If $\left[f_{1}, \ldots, f_{n}\right]$ is the set of Whitehead products, $W(\varphi)$, where $\varphi$ is of type $\left(f_{1}, \ldots, f_{n}\right)$, then WP $(X)=n$ if and only if $\left[f_{1}, \ldots, f_{n-1}\right]=0$ for all $\left(f_{1}, \ldots, f_{n-1}\right)$ and there is $\varphi: T_{1}\left(\Sigma Y_{1}, \ldots, \Sigma Y_{n}\right) \rightarrow X$ such that $W(\varphi) \neq 0$. wp $(X)=n$ if $0 \in\left[f_{1}, \ldots, f_{n-1}\right]$ for all $\left[f_{1}, \ldots, f_{n-1}\right]$ and there are $g_{1}, \ldots, g_{n}$ such that $0 \notin\left[g_{1}, \ldots, g_{n}\right]$.

wp $(X)=\mathrm{WP}(X)$ if wp $(X)<4$. We do not know if this is true in general $\left({ }^{4}\right)$.

Let $\lambda: \Sigma \Omega X \rightarrow X$ be the adjoint of the identity.

Proposition 6.13. wp $(X)=n$ if and only if $0 \in[\lambda, \ldots, \lambda](n-1)$ times and $0 \notin[\lambda, \ldots, \lambda] n$ times.

Proof. Given $f: \Sigma Y \rightarrow X$ there is $\bar{f}: \Sigma Y \rightarrow \Sigma \Omega X$ such that $\lambda \bar{f}=f$. Thus if there exists a map $\varphi:(\Sigma \Omega X)^{n-1} \rightarrow X$ of type $(\lambda)^{n-1}, \varphi\left(\bar{f}_{1} \times \cdots \times \bar{f}_{n-1}\right)$ is a map of type $\left(f_{1}, \ldots, f_{n-1}\right)$.

Definition 6.14. Define integers SC $(X), \operatorname{sc}(X)$, WC $(X)$ and wc $(X)$ by

(a) $\mathrm{SC}(X)$ is the least integer $n$ such that $\theta_{n}$ is essential.

(b) $\operatorname{sc}(X)$ is the least integer $n$ such that there exists $\varphi: Y_{1} \times \cdots \times Y_{n} \rightarrow E_{n}^{n}(X)$ for some $Y_{1}, \ldots, Y_{n}$ with the property that $q\left(\varphi^{*}\left[\theta_{n}\right]\right) \neq 0$. (Note that $\varphi$ need not be special.)

(c) $\mathrm{WC}(X)$ is the least integer $n$ such that there is a special map

$$
\varphi: Y_{1} \times \cdots \times Y_{n} \rightarrow E_{n}^{n}(X)
$$

for some spaces $Y_{1}, \ldots, Y_{n}$ with the property that $S_{n}(\varphi) \neq 0$.

(d) wc $(X)$ is the least integer $n$ such that there are maps $f_{1}, \ldots, f_{n}$ with the property that $0 \notin\left\langle f_{1}, \ldots, f_{n}\right\rangle$.

Proposition 6.15. If $X$ is an H-space

$$
\mathrm{SC}(X)=\operatorname{sc}(X)=\mathrm{wc}(X)=\mathrm{wC}(X)=\infty .
$$

Proposition 6.16. SC $(X) \leqq \mathrm{sc}(X) \leqq \mathrm{WC}(X) \leqq \mathrm{wc}(X)$.

Proposition 6.17. WC $(X)=\mathrm{WP}(X) ; \mathrm{wc}(X)=\mathrm{wp}(X)\left({ }^{4}\right)$.

It follows from Proposition 6.13 that $\operatorname{wc}(X)=n$ if there are liftings $\varphi_{j}:(\Omega X)^{j}$ $\rightarrow E_{j}^{j+1}(X)$ of type $(1, \ldots, 1)$ for $j<n$ but not for $j=n$. These liftings are given by maps $\tilde{\varphi}_{j}:(\Omega X)^{j} \rightarrow P \Omega^{j-1} X$ and hence by maps $\bar{\varphi}_{j}:(\Omega X)^{j} \times I^{j} \rightarrow X, j=2, \ldots$, $n-1$. It seems likely that these maps are essentially the maps $Q_{j}:(\Omega X)^{j} \times I^{j-1} \rightarrow \Omega X$ used by Williams [10] to define $C_{n}$ forms.

\section{REFERENCES}

1. H. H. Gershenson, Higher composition products, J. Math Kyoto Univ. 5 (1965), 1-37. MR 32 \#8341.

$\left.{ }^{4}\right)$ Added in proof. F. D. Williams has recently shown that wp $(X)=\mathrm{WP}(X)$ and hence wc $(X)=\mathrm{WC}(X)$. 
2. K. A. Hardie, Derived homotopy constructions, J. London Math. Soc. 35 (1960), 465-480. MR 23 \#A2883.

3. D. Kraines, Massey higher products, Trans. Amer. Math. Soc. 124 (1966), 431-449. MR 34 \#2010.

4. W. S. Massey, Some higher order cohomology operations, Symposium International de Topología Algebraica, Universidad Nacional Autónoma de México and UNESCO, Mexico City, 1958, pp. 145-154. MR 20 \#4826.

5. G. J. Porter, Higher-order Whitehead products, Topology 3 (1965), 123-135. MR 30 \#4261.

6. - Spaces with vanishing Whitehead products, Quart. J. Math. Oxford Ser. (2) 16 (1965), 77-84. MR 30 \#2511.

7. E. Spanier, Higher order operations, Trans. Amer. Math. Soc. 109 (1963), 509-539. MR 28 \#1622.

8. J. D. Stasheff, Homotopy associativity of H-spaces. I, Trans. Amer. Math. Soc. 108 (1963), 275-292. MR 28 \#1623.

9. H. Toda, Generalized Whitehead products and homotopy groups of spheres, J. Inst. Polytech. Osaka City Univ. Ser. A Math. 3 (1952), 43-82. MR 15, 732.

10. F. D. Williams, $A$ characterization of spaces with vanishing generalized higher Whitehead products, Bull. Amer. Math. Soc. 74 (1968), 497-499. MR 36 \#5944.

11. J. M. Cohen, The decomposition of stable homotopy, Ann. of Math. (2) 87 (1968), 305-320. MR 37 \#6932.

12. J. P. May, Matric Massey products, J. Algebra 12 (1969), 533-568. MR 39 \#289.

\section{University of Pennsylvania}

Philadelphia, Pennsylvania 19104 